\title{
Quenching Time Optimal Control for Some Ordinary Differential Equations
}

\author{
Ping Lin \\ School of Mathematics \& Statistics, Northeast Normal University, Changchun 130024, China \\ Correspondence should be addressed to Ping Lin; linp258@nenu.edu.cn
}

Received 23 January 2014; Accepted 28 February 2014; Published 10 April 2014

Academic Editor: Alberto Cabada

Copyright (C) 2014 Ping Lin. This is an open access article distributed under the Creative Commons Attribution License, which permits unrestricted use, distribution, and reproduction in any medium, provided the original work is properly cited.

This paper concerns time optimal control problems of three different ordinary differential equations in $\mathbb{R}^{2}$. Corresponding to certain initial data and controls, the solutions of the systems quench at finite time. The goal to control the systems is to minimize the quenching time. The purpose of this study is to obtain the existence and the Pontryagin maximum principle of optimal controls. The methods used in this paper adapt to more general and complex ordinary differential control systems with quenching property. We also wish that our results could be extended to the same issue for parabolic equations.

\section{Introduction}

In this paper, we study some quenching time optimal control problems of three different ordinary differential equations in $\mathbb{R}^{2}$. First of all, some notations will be introduced. We use $\|\cdot\|$ and $\langle\cdot, \cdot\rangle$ to stand for the Euclidean norm and the inner product of $\mathbb{R}^{2}$. For each matrix $D$, we use $D^{T}$ and $\|D\|$ to denote its transposition and the operator norm, respectively. Let $B(\cdot) \triangleq\left(\begin{array}{l}b_{11}(\cdot) b_{12}(\cdot) \\ b_{21}(\cdot)\end{array} b_{22} \cdot(\cdot)\right)$ be a nontrivial matrix-value function in the space $L^{\infty}\left(0,+\infty ; \mathbb{R}^{2 \times 2}\right)$. Write $R^{+}$for $[0,+\infty)$. For each $\rho_{0}>0$ given, we set

$$
\begin{gathered}
\mathcal{U}_{a d}=\left\{u: R^{+} \longrightarrow \mathbb{R}^{2} ; u\right. \text { is Lebesgue measurable, } \\
\left.\|u(t)\| \leq \rho_{0} \text { for a.e. } t \in R^{+}\right\} .
\end{gathered}
$$

Each $u(\cdot) \in \mathscr{U}_{\text {ad }}$ can be expressed as $u(\cdot)=\left(u_{1}(\cdot), u_{2}(\cdot)\right)^{T}$. Let

$$
\begin{array}{ll}
b_{1}(\cdot, u(\cdot)) \triangleq b_{11}(\cdot) u_{1}(\cdot)+b_{12}(\cdot) u_{2}(\cdot), & \text { when } u(\cdot) \in \mathscr{U}_{a d}, \\
b_{2}(\cdot, u(\cdot)) \triangleq b_{21}(\cdot) u_{1}(\cdot)+b_{22}(\cdot) u_{2}(\cdot), & \text { when } u(\cdot) \in \mathscr{u}_{a d} .
\end{array}
$$

For each $C^{1}$-function $g: E \subset \mathbb{R}^{2} \rightarrow \mathbb{R}^{2}$, its derivative will be written as

$$
\left(\begin{array}{ll}
\frac{\partial g_{1}}{\partial y_{1}} & \frac{\partial g_{2}}{\partial y_{1}} \\
\frac{\partial g_{1}}{\partial y_{2}} & \frac{\partial g_{2}}{\partial y_{2}}
\end{array}\right)
$$

where $g=\left(g_{1}, g_{2}\right)^{T}$ with $g_{i}: E \subset \mathbb{R}^{2} \rightarrow \mathbb{R}^{1}, i=1,2$.

The controlled systems under consideration are as follows:

$$
\begin{gathered}
\frac{d y(t)}{d t}=f(y(t))+B(t) u(t), \quad t>0, \\
y(0)=y^{0} .
\end{gathered}
$$

Here, $y^{0}=\left(y_{1}^{0}, y_{2}^{0}\right)^{T} \in \mathbb{R}^{2}, u(\cdot) \in \mathcal{U}_{a d}$, and $f \in \Lambda \triangleq$ $\left\{f^{(1)}, f^{(2)}, f^{(3)}\right\}$, where

(i) $f^{(1)}=\left(f_{1}^{(1)}, f_{2}^{(1)}\right)^{T}: \mathbb{R}^{2} \backslash\left\{y=\left(y_{1}, y_{2}\right)^{T} \in \mathbb{R}^{2} ; y_{1}=\right.$ $1\} \rightarrow \mathbb{R}^{2}, f_{1}^{(1)}(y)=y_{2} /\left(1-y_{1}\right)$, and $f_{2}^{(1)}(y)=y_{1}+y_{2} ;$

(ii) $f^{(2)}=\left(f_{1}^{(2)}, f_{2}^{(2)}\right)^{T}: \mathbb{R}^{2} \backslash\left\{y=\left(y_{1}, y_{2}\right)^{T} \in \mathbb{R}^{2} ; y_{1}^{2}+\right.$ $\left.y_{2}^{2}=1\right\} \rightarrow \mathbb{R}^{2}, f_{1}^{(2)}(y)=y_{1} /\left(1-\sqrt{y_{1}^{2}+y_{2}^{2}}\right)$, and $f_{2}^{(2)}(y)=y_{2} /\left(1-\sqrt{y_{1}^{2}+y_{2}^{2}}\right)$; 
(iii) $f^{(3)}=\left(f_{1}^{(3)}, f_{2}^{(3)}\right)^{T}: \mathbb{R}^{2} \backslash\left\{y=\left(y_{1}, y_{2}\right)^{T} \in \mathbb{R}^{2} ; y_{1}=\right.$ 1 or $\left.y_{2}=1\right\} \rightarrow \mathbb{R}^{2}, f_{1}^{(3)}(y)=1 /\left(1-y_{2}\right)$, and $f_{2}^{(3)}(y)=1 /\left(1-y_{1}\right)$.

Let

$$
K_{0}=\underset{s \in R^{+}}{\operatorname{esssup}}\|B(s)\| \rho_{0} .
$$

Define

$$
\begin{aligned}
& S^{f^{(1)}=}\left\{\left(z_{1}, z_{2}\right) \in \mathbb{R}^{2} ; z_{1} \in\left(1-\frac{1}{2 K_{0}}, 1\right),\right. \\
&\left.z_{2} \in\left(K_{0}+\frac{1}{K_{0}}-1,+\infty\right)\right\} \\
& \cup\left\{\left(z_{1}, z_{2}\right) \in \mathbb{R}^{2} ; z_{1} \in\left(1,1+\frac{1}{2 K_{0}}\right),\right. \\
& S^{f^{(2)}}=\left\{\left(z_{1}, z_{2}\right) \in \mathbb{R}^{2} ;\|z\| \in\left(1-\frac{1}{2 K_{0}+1}, 1\right)\right\} \\
& \cup\left\{\left(z_{1}, z_{2}\right) \in \mathbb{R}^{2} ;\|z\| \in\left(1,1+\frac{1}{2 K_{0}}\right)\right\} ; \\
& S^{f^{(3)}}=\left\{\left(z_{1}, z_{2}\right) \in \mathbb{R}^{2} ; z_{1} \in\left(1-\frac{e^{-3 / 2}}{2 K_{0}}, 1\right),\right. \\
& \cup\left\{\left(z_{1}, z_{2}\right) \in \mathbb{R}^{2} ; z_{1} \in\left(1,1+\frac{e^{-3 / 2}}{2 K_{0}}\right),\right. \\
&\left.z_{2} \in\left(1-\frac{e^{-3 / 2}}{2 K_{0}}, 1\right)\right\} \\
&\left.\left.z^{-3 / 2}\right)\right\} .
\end{aligned}
$$

Since, for each $f \in \Lambda, f$ is continuously differential over the domain $S^{f}$, it is clear that, given $f \in \Lambda, y^{0} \in S^{f}$, and $u \in \mathcal{U}_{\text {ad }}$, the controlled system (4) has a unique solution. We denote this solution by $y\left(\cdot ; f, y^{0}, u\right)=\left(y_{1}\left(\cdot ; f, y^{0}, u\right), y_{2}\left(\cdot ; f, y^{0}, u\right)\right)^{T}$ and write $\left[0, T_{\max }\left(f, y^{0}, u\right)\right)$ for its maximal interval of existence.

It is shown in Section 2 that, given $f \in \Lambda, y^{0} \in S^{f}$, and $u \in \mathcal{U}_{a d}$, there exists a time $T_{q}\left(f, y^{0}, u\right)$ with $T_{q}\left(f, y^{0}, u\right) \leq$ $T_{\max }\left(f, y^{0}, u\right)$ holding the property that

$$
\begin{gathered}
0<T_{q}\left(f, y^{0}, u\right)<+\infty, \\
\lim _{t \rightarrow T_{q}\left(f, y^{0}, u\right)}\left\|f\left(y\left(t ; f, y^{0}, u\right)\right)\right\|=+\infty, \\
\left\|f\left(y\left(t ; f, y^{0}, u\right)\right)\right\|<+\infty \quad \text { as } t \in\left[0, T_{q}\left(f, y^{0}, u\right)\right) .
\end{gathered}
$$

We say that the solution $y\left(\cdot ; f, y^{0}, u\right)$ quenches at the finite time $T_{q}\left(f, y^{0}, u\right)$ and $T_{q}\left(f, y^{0}, u\right)$ is the quenching time of the solution $y\left(\cdot ; f, y^{0}, u\right)$.
The purpose of this paper is to study the existence and the Pontryagin maximum principle for the following time optimal control problems:

$$
\min _{u \in \mathscr{U}_{a d}}\left\{T_{q}\left(f, y^{0}, u\right)\right\}, \quad \text { where } f \in \Lambda, y^{0} \in S^{f} .
$$

Because of (7), for each $f \in \Lambda$ and $y^{0} \in S^{f}$, there exists a number $t^{*}\left(f, y^{0}\right)$ in $R^{+}$such that

$$
t^{*}\left(f, y^{0}\right)=\inf _{u \in \mathcal{U}_{a d}} T_{q}\left(f, y^{0}, u\right),
$$

which is called the optimal time for the problem $(P)_{y^{0}}^{f}$. A control $u^{*}(\cdot)$, in the set $\mathcal{U}_{a d}$ holding the property: $T_{q}\left(f, y^{0}, u^{*}\right)=$ $t^{*}\left(f, y^{0}\right)$, is called an optimal control, while the solution $y\left(\cdot ; f, y^{0}, u^{*}\right)$ is called the optimal state corresponding to $u^{*}$ for the problem $(P)_{y^{0}}^{f}$. We will simply write $y^{*}(\cdot)$ for the optimal state $y\left(\cdot ; f, y^{0}, u^{*}\right)$.

The main results of this paper are as follows.

Theorem 1. Given $i \in\{1,2,3\}$ and $y^{0} \in S^{f^{(i)}}$, the problem $(P)_{y^{0}}^{f^{(i)}}$ has optimal controls.

Theorem 2. Let $y^{0} \in S^{f^{(1)}}$. Then, Pontryagin's maximum principle holds for the problem $(P)_{y^{0}}^{f^{(1)}}$. Namely, if $t^{*}$ is the optimal time, $u^{*}$ is an optimal control, and $y^{*}$ is the corresponding optimal state for the problem $(P)_{y^{0}}^{f^{(1)}}$, then there is a nontrivial function $\psi(\cdot)$ in the space $C\left(\left[0, t^{*}\right] ; \mathbb{R}^{2}\right)$ satisfying

$$
\begin{aligned}
\psi(t)=\int_{t}^{t^{*}} f_{y}^{(1)}\left(y^{*}(\tau)\right) \psi(\tau) d \tau \quad \forall t \in\left[0, t^{*}\right), \\
\psi\left(t^{*}\right)=0, \\
\max _{\|u\| \leq \rho_{0}}\langle\psi(t), B(t) u\rangle=\left\langle\psi(t), B(t) u^{*}(t)\right\rangle
\end{aligned}
$$

$$
\text { for a.e. } t \in\left[0, t^{*}\right] \text {. }
$$

Besides, it holds that

$$
t^{*} \leq\left(y_{1}^{0}-1\right)^{2}
$$

Theorem 3. Let $y^{0} \in S^{f^{(2)}}$. Then, Pontryagin's maximum principle holds for the problem $(P)_{y^{0}}^{f^{(2)}}$. Namely, if $t^{*}$ is the optimal time, $u^{*}$ is an optimal control, and $y^{*}$ is the corresponding optimal state for the problem $(P)_{y^{0}}^{f^{(2)}}$, then there is a nontrivial function $\psi(\cdot)$ in the space $C\left(\left[0, t^{*}\right] ; \mathbb{R}^{2}\right)$ satisfying

$$
\begin{array}{r}
\psi(t)=\int_{t}^{t^{*}} f_{y}^{(2)}\left(y^{*}(\tau)\right) \psi(\tau) d \tau \quad \forall t \in\left[0, t^{*}\right), \\
\psi\left(t^{*}\right)=0, \\
\max _{\|u\| \leq \rho_{0}}\langle\psi(t), B(t) u\rangle=\left\langle\psi(t), B(t) u^{*}(t)\right\rangle \\
\text { for a.e. } t \in\left[0, t^{*}\right] .
\end{array}
$$


Besides, it holds that

$$
t^{*} \leq \frac{\left(2 K_{0}+1\right)\left(\left\|y^{0}\right\|-1\right)^{2}}{2 K_{0}} .
$$

It is worth mentioning that problem $(P)_{y^{0}}^{f^{(3)}}$ is more complicated than the other two problems since the target set of problem $(P)_{y^{0}}^{f^{(3)}}$ is more complicated than the target sets of the other two problems. Indeed, the target set of problem $(P)_{y^{0}}^{f^{(3)}}$ is $\left\{y \in \mathbb{R}^{2} ; y_{1}=1\right.$ or $\left.y_{2}=1\right\}$, while the target sets for problem $(P)_{y^{0}}^{f^{(1)}}$ and problem $(P)_{y^{0}}^{f^{(2)}}$ are $\left\{y \in \mathbb{R}^{2} ; y_{1}=1\right\}$ and $\left\{y \in \mathbb{R}^{2} ;\|y\|=1\right\}$, respectively. This is why we only obtain the existence but not Pontryagin's maximum principle for problem $(P)_{y^{0}}^{f^{(3)}}$.

The concept of quenching was first introduced by Kawarada in [1] for a nonlinear parabolic equation; it has more general sense than blowup. For instance, consider the equation $d y(t) / d t=-1 /\left(\sin ^{2}(y(t)) \cos (y(t))\right), y(0)=\pi / 6$. It is obvious that the first quenching time of the solution for this equation is $1 / 24$ at which $\sin (y(1 / 24))=0$. Then the solution $y(\cdot)$ can be extended, for instance, until $y(1 / 12)=$ $-\pi / 6$. Further, the solution $y(\cdot)$ can be extended again until $\cos (y(3 / 8))=0$; that is, the second quenching time is $3 / 8$. This differs from the blowup ordinary differential equations, where the solutions tend to infinity at blowup time.

There have been many literatures concerning the properties of parabolic differential equations with quenching behavior (see $[1,2]$ and references therein). To the best of our knowledge, the study on quenching time optimal control problems has not been touched upon. In this paper, we focus on quenching time optimal control problems for ordinary differential equations with three particular vector fields $f^{(i)}$, $i=1,2,3$, in $\mathbb{R}^{2}$. Indeed, the methods used in this paper adapt to more general and complex ordinary differential control systems with quenching property. For instance, we can use the similar methods we use in this paper to consider the quenching time optimal problem for system (4), where $f_{1}(y)=-1 /\left(\sin ^{2} y \cos y\right), f_{2}(y)=y_{1}+y_{2}$ (we will give the details in the following section). We also wish that our results could be extended to the same issue for parabolic equations.

The differential systems whose solutions have the behavior of quenching arise in the study of chemical reactions. Quenching phenomena could describe the completion of a chemical reaction. Using catalysts to make a chemical reaction complete in the shortest time could be considered an optimal quenching time problem. It is also significant in the theory studies of the electric current transient phenomena in polarized ionic conductors. In certain cases, the quenching of a solution is desirable. Thus, it could be interesting to minimize the quenching time with the aid of controls in certain cases. It deserves to mention that the quenching time $T_{q}\left(f, y^{0}, u\right)$, with some control $u$, can really be strictly less than $T_{q}\left(f, y^{0}, 0\right)$. Here is an example. Consider the problem $(P)_{y^{0}}^{f^{(2)}}$, where $y_{1}^{0}=3 / 4, y_{2}^{0}=0$ and $B(t)=\left(\begin{array}{ll}1 & 0 \\ 0 & 0\end{array}\right)$ for all $t \in R^{+}$.
It can be directly checked that $T_{q}\left(f^{(2)},(3 / 4,0)^{T},(1,0)^{T}\right)=$ $1 / 32<T_{q}\left(f^{(2)},(3 / 4,0)^{T}, 0\right)=-1 / 4-\ln (3 / 4)$.

Because of the quenching behavior of solutions to system (4), the usual methods applied to solve the general optimal control problems (see, for instance, [3-7]) do not work for problem $(P)_{y^{0}}^{f}$ with $f \in \Lambda$ and $y^{0} \in S^{f}$. We approach our main results by the following steps. First, we show some invariant properties for solutions of system (4). Next, we prove that, given $f \in \Lambda$ and $y^{0} \in S^{f}$, the corresponding solution of system (4) quenches at finite time for each control $u \in \mathcal{U}_{a d}$. Then we give the quenching rate estimate for solutions of system (4). Furthermore, we obtain the following property. When a sequence $\left\{u_{k}\right\}$ of controls tends to a control $u$ in a suitable topology, the solutions $y\left(\cdot ; f, y^{0}, u_{k}\right)$ with $k$ sufficiently large share a common interval of nonquenching with the solution $y\left(\cdot ; f, y^{0}, u\right)$. Finally, we use the abovementioned results to verify our main theorems.

Since blowup could be regarded as a special quenching phenomenon, it deserves to mention the paper [8], where minimal blowup time optimal control problems of ordinary differential equations were studied. Inspired by the idea in [8], we develop new methods in this paper.

The rest of the paper is organized as follows. Section 2 presents some preliminary lemmas which supply some properties of solutions to controlled system (4). Section 3 proves the existence of optimal controls for problem $(P)_{y^{0}}^{f}$ with $f \in$ $\Lambda$ and $y^{0} \in S^{f}$. Section 4 verifies Theorems 2 and 3 .

\section{Preliminary Lemmas}

In this section, we will introduce some properties of solutions to system (4), which will play important roles to prove our main results.

2.1. The Existence and Invariant Property. Consider the system

$$
\begin{gathered}
\frac{d \xi(t)}{d t}=f(\xi(t))+B(t) u(t), \quad t>t_{0}, \\
\xi\left(t_{0}\right)=y^{0} .
\end{gathered}
$$

Since, for each $f \in \Lambda, f$ is continuously differential over the domain $S^{f}$, it is clear that, given $t_{0} \geq 0, f \in \Lambda, y^{0} \in S^{f}$, and $u \in \mathscr{U}_{a d}$, system (15) has a unique solution. We denote this solution by $\xi\left(\cdot ; t_{0}, f, y^{0}, u\right)=\left(\xi_{1}\left(\cdot ; t_{0}, f, y^{0}, u\right)\right.$, $\left.\xi_{2}\left(\cdot ; t_{0}, f, y^{0}, u\right)\right)^{T}$ and write $\left[t_{0}, T_{\max }\left(t_{0}, f, y^{0}, u\right)\right)$ for its maximal interval of existence.

Let $t_{0} \geq 0, y^{0}=\left(y_{1}^{0}, y_{2}^{0}\right)^{T} \in S^{f^{(1)}}$ and $u \in \mathscr{U}_{a d}$. If $y_{1}^{0}<$ 1 ; then, by the continuity of the solution $\xi\left(\cdot ; t_{0}, f^{(1)}, y^{0}, u\right)$, we can find a positive number $\alpha$ sufficiently small such that $\xi_{1}\left(t ; t_{0}, f^{(1)}, y^{0}, u\right)<1$ for all $t \in\left[t_{0}, t_{0}+\alpha\right)$. Similarly, if $y_{1}^{0}>1$, we can find a positive number $\beta$ sufficiently small such that $\xi_{1}\left(t ; t_{0}, f^{(1)}, y^{0}, u\right)>1$ for all $t \in\left[t_{0}, t_{0}+\right.$ $\beta)$. Given $t_{0} \geq 0, y^{0}=\left(y_{1}^{0}, y_{2}^{0}\right)^{T} \in S^{f^{(1)}}$, and $u \in$ $\mathcal{U}_{a d}$, we will use the notation $I\left(t_{0}, f^{(1)}, y^{0}, u\right)$ to denote such 
a subinterval of the interval $\left[t_{0}, T_{\max }\left(t_{0}, f^{(1)}, y^{0}, u\right)\right)$. When $y_{1}^{0}<1, I\left(t_{0}, f^{(1)}, y^{0}, u\right)$ denotes the maximal time interval in which $\xi_{1}\left(t ; t_{0}, f^{(1)}, y^{0}, u\right)<1$, while, when $y_{1}^{0}>1$, $I\left(t_{0}, f^{(1)}, y^{0}, u\right)$ denotes the maximal time interval in which $\xi_{1}\left(t ; t_{0}, f^{(1)}, y^{0}, u\right)>1$. By the continuity of the solution $\xi\left(\cdot ; t_{0}, f^{(1)}, y^{0}, u\right)$ again, it is clear from the existence theorem and the extension theorem of ordinary differential equations that $I\left(t_{0}, f^{(1)}, y^{0}, u\right)$ is a left closed and right open interval, whose left end point is $t_{0}$. Let $1-1 /\left(2 K_{0}\right)<K_{1}<1$, $1<\widetilde{K}_{1}<1+1 /\left(2 K_{0}\right), K_{2}>K_{0}+1 / K_{0}-1$, and $\widetilde{K}_{2}>K_{0}+1$.

We have the following lemma.

Lemma 4. Given $t_{0} \geq 0, y^{0} \in S^{f^{(1)}}$, and $u \in \mathcal{U}_{a d}$, then, for all $t \in I\left(t_{0}, f^{(1)}, y^{0}, u\right)$

$$
\begin{array}{r}
\xi_{1}\left(t ; t_{0}, f^{(1)}, y^{0}, u\right) \geq K_{1}, \quad \xi_{2}\left(t ; t_{0}, f^{(1)}, y^{0}, u\right) \geq K_{2}, \\
\text { as } K_{1} \leq y_{1}^{0}<1, \quad y_{2}^{0} \geq K_{2} ; \\
\xi_{1}\left(t ; t_{0}, f^{(1)}, y^{0}, u\right) \leq \widetilde{K}_{1}, \quad \xi_{2}\left(t ; t_{0}, f^{(1)}, y^{0}, u\right) \geq \widetilde{K}_{2}, \\
\text { as } 1<y_{1}^{0} \leq \widetilde{K}_{1}, \quad y_{2}^{0} \geq \widetilde{K}_{2} .
\end{array}
$$

Proof. First, we claim the following property (A). Suppose that $t_{0} \geq 0, y^{0} \in S^{f^{(1)}}$, with $K_{1} \leq y_{1}^{0}<1, y_{2}^{0} \geq K_{2}$, and $u \in \mathscr{u}_{a d}$. Then, there is a positive number $\eta$ with $\left[t_{0}, t_{0}+\right.$ $\eta) \subset I\left(t_{0}, f^{(1)}, y^{0}, u\right)$ such that the solution $\xi\left(\cdot ; t_{0}, f^{(1)}, y^{0}, u\right)$ holds the following inequalities: $\xi_{1}\left(t ; t_{0}, f^{(1)}, y^{0}, u\right) \geq K_{1}$ and $\xi_{2}\left(t ; t_{0}, f^{(1)}, y^{0}, u\right) \geq K_{2}$ for all $t$ in the interval $\left[t_{0}, t_{0}+\eta\right]$.

Indeed, since $1-1 /\left(2 K_{0}\right)<K_{1} \leq y_{1}^{0}<1$ and $y_{2}^{0} \geq K_{2}>K_{0}+1 / K_{0}-1$, we can use the continuity of the solution $\xi\left(\cdot ; t_{0}, f^{(1)}, y^{0}, u\right)$ to find a positive constant $\eta$ such that $\left[t_{0}, t_{0}+\eta\right) \subset I\left(t_{0}, f^{(1)}, y^{0}, u\right), 1-1 /\left(2 K_{0}\right)<$ $\xi_{1}\left(t ; t_{0}, f^{(1)}, y^{0}, u\right)<1$, and $\xi_{2}\left(t ; t_{0}, f^{(1)}, y^{0}, u\right)>K_{0}+1 / K_{0}-1$ for all $t \in\left[t_{0}, t_{0}+\eta\right]$. Hence, it follows from (15) with $f=$ $f^{(1)},(5)$, and the inequality $K_{0}+1 / K_{0}-1 \geq 1$ that, for all $t \in\left[t_{0}, t_{0}+\eta\right]$,

$$
\begin{aligned}
\xi_{1}(t)-y_{1}^{0} & =\int_{t_{0}}^{t}\left(\frac{\xi_{2}(\tau)}{1-\xi_{1}(\tau)}+b_{1}(\tau, u(\tau))\right) d \tau \\
& \geq \int_{t_{0}}^{t}\left(\frac{K_{0}+1 / K_{0}-1}{1 /\left(2 K_{0}\right)}-K_{0}\right) d \tau \geq 0 \\
\xi_{2}(t)-y_{2}^{0} & =\int_{t_{0}}^{t}\left(\xi_{1}(\tau)+\xi_{2}(\tau)+b_{2}(\tau, u(\tau))\right) d \tau \\
& \geq \int_{t_{0}}^{t}\left(1-\frac{1}{2 K_{0}}+\frac{1}{K_{0}}-1\right) d \tau \geq 0
\end{aligned}
$$

Here, we simply write $\xi(\cdot)$ for the solution $\xi\left(\cdot ; t_{0}, f^{(1)}, y^{0}, u\right)$. From the two inequalities mentioned above and the inequalities $y_{1}^{0} \geq K_{1}$ and $y_{2}^{0} \geq K_{2}$, we get the property (A).

Now, we come back to prove the property (16).
By seeking a contradiction, suppose that there exist a time $t_{0} \geq 0$, initial data $y^{0} \in S^{f^{(1)}}$, a control $u \in \mathcal{U}_{\text {ad }}$, and a number pair $\left(K_{1}, K_{2}\right)$ with $1-1 /\left(2 K_{0}\right)<K_{1}<1$ and $K_{2}>K_{0}+1 / K_{0}-1$ such that the solution $\xi\left(\cdot ; t_{0}, f^{(1)}, y^{0}, u\right)$ with $K_{1} \leq y_{1}^{0}<1$ and $y_{2}^{0} \geq K_{2}$ does not satisfy (16). Then we would find a number $s_{0}>t_{0}$ in the interval $I\left(t_{0}, f^{(1)}, y^{0}, u\right)$ such that $\xi_{1}\left(s_{0} ; t_{0}, f^{(1)}, y^{0}, u\right)<K_{1}$ or $\xi_{2}\left(s_{0} ; t_{0}, f^{(1)}, y^{0}, u\right)<K_{2}$. Write $s_{1}$ for $\inf \left\{t \in\left[t_{0}, s_{0}\right] ; \xi_{1}\left(t ; t_{0}, f^{(1)}, y^{0}, u\right)<K_{1}\right\}$ and write $s_{2}$ for $\inf \left\{t \in\left[t_{0}, s_{0}\right] ; \xi_{2}\left(t ; t_{0}, f^{(1)}, y^{0}, u\right)<K_{2}\right\}$. We may as well assume that $s_{1} \leq s_{2}$. Since $\xi\left(\cdot ; t_{0}, f^{(1)}, y^{0}, u\right)$ is continuous over $I\left(t_{0}, f^{(1)}, y^{0}, u\right)$, we can use the definition of $s_{1}$ and $s_{2}$ and the inequality $s_{1} \leq s_{2}$ to derive the following properties: (a) $\xi_{1}\left(s_{1} ; t_{0}, f^{(1)}, y^{0}, u\right) \geq K_{1}$ and (b) corresponding to each $\delta$ with $\left(s_{1}, s_{1}+\delta\right) \subset I\left(t_{0}, f^{(1)}, y^{0}, u\right)$, there exists a number $t_{\delta}$ in $\left(s_{1}, s_{1}+\delta\right)$ such that $\xi_{1}\left(t_{\delta} ; t_{0}, f^{(1)}, y^{0}, u\right)<K_{1}$; (c) $\xi_{2}\left(t ; t_{0}, f^{(1)}, y^{0}, u\right) \geq K_{2}$, for each $t \in\left[t_{0}, s_{1}\right]$. Write $z=\xi\left(s_{1} ; t_{0}, f^{(1)}, y^{0}, u\right)$. Then, it follows from the properties (a), (c), and the definition of the interval $I\left(t_{0}, f^{(1)}, y^{0}, u\right)$ that $K_{1} \leq z_{1}=\xi_{1}\left(s_{1} ; t_{0}, f^{(1)}, y^{0}, u\right)<1$ and $z_{2}=$ $\xi_{2}\left(s_{1} ; t_{0}, f^{(1)}, y^{0}, u\right) \geq K_{2}$. Consider the following system:

$$
\begin{gathered}
\frac{d \xi(t)}{d t}=f(\xi(t))+B(t) u(t), \quad t>s_{1}, \\
\xi\left(s_{1}\right)=z .
\end{gathered}
$$

Making use of the property (A), we can choose a positive number $\delta_{0}$ sufficiently small such that

$$
\begin{array}{r}
\xi_{1}\left(t ; s_{1}, f^{(1)}, z, u\right) \geq K_{1}, \quad \xi_{2}\left(t ; s_{1}, f^{(1)}, z, u\right) \geq K_{2} \\
\text { for each } t \in\left[s_{1}, s_{1}+\delta_{0}\right) .
\end{array}
$$

On the other hand, the function $\xi\left(\cdot ; t_{0}, f^{(1)}, y^{0}, u\right)$ also satisfies the system (19) when $t \geq s_{1}$. By the uniqueness of the solution to the system (19), we necessarily have $\xi\left(t ; t_{0}, f^{(1)}, y^{0}, u\right)=\xi\left(t ; s_{1}, f^{(1)}, z, u\right)$ for all $t \geq s_{1}$. This, combined with the above-mentioned property (b), implies the existence of a number $t_{\delta_{0}}$ in $\left(s_{1}, s_{1}+\delta_{0}\right)$ such that $\xi_{1}\left(t_{\delta_{0}} ; s_{1}, f^{(1)}, z, u\right)<K_{1}$. This contradicts to (20). Therefore, we have accomplished the proof of the property (16).

We can use the very similar argument of the proof of (16) to prove (17) (see [9] for the detailed proofs).

The following two lemmas play important roles in the proof of Theorem 1 with $f=f^{(2)}$ and $f=f^{(3)}$ and Theorem 3 (see [9] for the detailed proofs).

Let $t_{0} \geq 0, y^{0} \in S^{f^{(2)}}$, and $u \in \mathcal{U}_{a d}$; we will use the notation $I\left(t_{0}, f^{(2)}, y^{0}, u\right)$ to denote such a subinterval of the interval $\left[t_{0}, T_{\max }\left(t_{0}, f^{(2)}, y^{0}, u\right)\right)$. When $\left\|y^{0}\right\|<$ $1, I\left(t_{0}, f^{(2)}, y^{0}, u\right)$ denotes the maximal time interval in which $\left\|\xi\left(t ; t_{0}, f^{(2)}, y^{0}, u\right)\right\|<1$, while, when $\left\|y^{0}\right\|>1$, $I\left(t_{0}, f^{(2)}, y^{0}, u\right)$ denotes the maximal time interval in which $\left\|\xi\left(t ; t_{0}, f^{(2)}, y^{0}, u\right)\right\|>1$. It is clear that $I\left(t_{0}, f^{(2)}, y^{0}, u\right)$ is a left closed and right open interval, whose left end point is $t_{0}$. Let $1-1 /\left(2 K_{0}+1\right)<K_{3}<1$ and $1<\widetilde{K}_{3}<1+1 /\left(2 K_{0}\right)$.

We have the following lemma. 
Lemma 5. Given $t_{0} \geq 0, y^{0} \in S^{f^{(2)}}$, and $u \in \mathcal{U}_{\text {ad }}$, then, for all $t \in I\left(t_{0}, f^{(2)}, y^{0}, u\right)$

$$
\begin{aligned}
& \left\|\xi\left(t ; t_{0}, f^{(2)}, y^{0}, u\right)\right\| \geq K_{3}, \quad \text { when } K_{3} \leq\left\|y^{0}\right\|<1 ; \\
& \left\|\xi\left(t ; t_{0}, f^{(2)}, y^{0}, u\right)\right\| \leq \widetilde{K}_{3}, \quad \text { when } 1<\left\|y^{0}\right\| \leq \widetilde{K}_{3} .
\end{aligned}
$$

Let $t_{0} \geq 0, y^{0} \in S^{f^{(3)}}$, and $u \in \mathcal{U}_{a d}$; we will use the notation $I\left(t_{0}, f^{(3)}, y^{0}, u\right)$ to denote such a subinterval of the interval $\left[t_{0}, T_{\max }\left(t_{0}, f^{(3)}, y^{0}, u\right)\right)$. When $y_{1}^{0}<1$ and $y_{2}^{0}<1$, $I\left(t_{0}, f^{(3)}, y^{0}, u\right)$ denotes the maximal time interval in which $\xi_{1}\left(t ; t_{0}, f^{(3)}, y^{0}, u\right)<1$ and $\xi_{2}\left(t ; t_{0}, f^{(3)}, y^{0}, u\right)<1$, while, when $y_{1}^{0}>1$ and $y_{2}^{0}>1, I\left(t_{0}, f^{(3)}, y^{0}, u\right)$ denotes the maximal time interval in which $\xi_{1}\left(t ; t_{0}, f^{(3)}, y^{0}, u\right)>1$ and $\xi_{2}\left(t ; t_{0}, f^{(3)}, y^{0}, u\right)>1$. It is clear that $I\left(t_{0}, f^{(3)}, y^{0}, u\right)$ is a left closed and right open interval, whose left end point is $t_{0}$. Let $1-e^{-3 / 2} /\left(2 K_{0}\right)<K_{4}<1$ and $1<\widetilde{K}_{4}<1+e^{-3 / 2} /\left(2 K_{0}\right)$.

We have the following lemma.

Lemma 6. Given $t_{0} \geq 0, y^{0} \in S^{f^{(3)}}$, and $u \in \mathcal{U}_{a d}$, then, for all $t \in I\left(t_{0}, f^{(3)}, y^{0}, u\right)$,

$$
\begin{array}{r}
\xi_{1}\left(t ; t_{0}, f^{(3)}, y^{0}, u\right) \geq K_{4}, \quad \xi_{2}\left(t ; t_{0}, f^{(3)}, y^{0}, u\right) \geq K_{4}, \\
\text { when } K_{4} \leq y_{1}^{0}<1, \quad K_{4} \leq y_{2}^{0}<1 ; \\
\xi_{1}\left(t ; t_{0}, f^{(3)}, y^{0}, u\right) \leq \widetilde{K}_{4}, \quad \xi_{2}\left(t ; t_{0}, f^{(3)}, y^{0}, u\right) \leq \widetilde{K}_{4}, \\
\text { when } 1<y_{1}^{0} \leq \widetilde{K}_{4}, \quad 1<y_{2}^{0} \leq \widetilde{K}_{4} .
\end{array}
$$

\subsection{Quenching Property and Estimate of Quenching Rate}

Lemma 7. For each $y^{0} \in S^{f^{(1)}}$ and each $u \in \mathcal{U}_{a d}$, there exists a time $T_{q}\left(f^{(1)}, y^{0}, u\right)$ in the interval $\left(0, T_{\max }\left(f^{(1)}, y^{0}, u\right)\right]$ holding the property that

$$
\begin{gathered}
T_{q}\left(f^{(1)}, y^{0}, u\right) \leq\left(y_{1}^{0}-1\right)^{2}<+\infty, \\
\lim _{t \rightarrow T_{q}\left(f^{(1)}, y^{0}, u\right)} y_{1}\left(t ; f^{(1)}, y^{0}, u\right)=1, \\
\lim _{t \rightarrow T_{q}\left(f^{(1)}, y^{0}, u\right)}\left\|f^{(1)}\left(y\left(t ; f^{(1)}, y^{0}, u\right)\right)\right\|=+\infty, \\
\left\|f^{(1)}\left(y\left(t ; f^{(1)}, y^{0}, u\right)\right)\right\|<+\infty \\
\text { as } t \in\left[0, T_{q}\left(f^{(1)}, y^{0}, u\right)\right) .
\end{gathered}
$$

Moreover, there exists a positive constant $C$, independent of $y^{0} \in S^{f^{(1)}}$ and $u$, such that

$$
\begin{array}{r}
\frac{1}{\left|1-y_{1}\left(t ; f^{(1)}, y^{0}, u\right)\right|} \leq C\left(T_{q}\left(f^{(1)}, y^{0}, u\right)-t\right)^{-2 / 3}, \\
t \in\left[0, T_{q}\left(f^{(1)}, y^{0}, u\right)\right) .
\end{array}
$$

Proof. Suppose that $y^{0} \in S^{f^{(1)}}$ and $u \in \mathcal{U}_{a d}$. Let $T_{q}\left(f^{(1)}, y^{0}, u\right)$ be the right end point of the interval $I\left(0, f^{(1)}, y^{0}, u\right)$. Thus, $I\left(0, f^{(1)}, y^{0}, u\right)=\left[0, T_{q}\left(f^{(1)}, y^{0}, u\right)\right)$ and $T_{q}\left(f^{(1)}, y^{0}, u\right) \in$ $\left(0, T_{\max }\left(f^{(1)}, y^{0}, u\right)\right]$ (see the definition and the property of $I\left(0, f^{(1)}, y^{0}, u\right)$ on page 3$)$. We will prove Lemma 7 in the following two cases.

Case $1.1-1 /\left(2 K_{0}\right)<y_{1}^{0}<1$ and $y_{2}^{0}>K_{0}+1 / K_{0}-1$.

Step 1. This is to prove (23) in Case 1.

By property (16) in Lemma 4 and the definition of $I\left(0, f^{(1)}, y^{0}, u\right)$ and $T_{q}\left(f^{(1)}, y^{0}, u\right)$, the solution $y\left(\cdot ; f^{(1)}, y^{0}, u\right)$ with $1-1 /\left(2 K_{0}\right)<y_{1}^{0}<1, y_{2}^{0}>K_{0}+1 / K_{0}-1$, and $u \in \mathcal{U}_{a d}$ holds the property that, for each $t \in\left[0, T_{q}\left(f^{(1)}, y^{0}, u\right)\right)$,

$$
\begin{aligned}
& 1-\frac{1}{2 K_{0}}<y_{1}^{0} \leq y_{1}\left(t ; f^{(1)}, y^{0}, u\right)<1, \\
& y_{2}\left(t ; f^{(1)}, y^{0}, u\right) \geq y_{2}^{0}>K_{0}+\frac{1}{K_{0}}-1 .
\end{aligned}
$$

Then, from system (4) with $f=f^{(1)},(5)$, and the inequality $K_{0}+1 / K_{0}-1 \geq 1$, it holds that

$$
\begin{aligned}
\frac{d y_{1}(t)}{d t} & =\frac{y_{2}(t)}{2\left(1-y_{1}(t)\right)}+\frac{y_{2}(t)}{2\left(1-y_{1}(t)\right)}+b_{1}(t, u(t)) \\
& \geq \frac{1}{2\left(1-y_{1}(t)\right)} \quad \text { for a.e. } t \in\left[0, T_{q}\left(f^{(1)}, y^{0}, u\right)\right) .
\end{aligned}
$$

Here and throughout the proof, we simply write $y(\cdot)$ for $y\left(\cdot ; f^{(1)}, y^{0}, u\right)$.

Let $\chi(\cdot)$ be the solution to the following equation:

$$
\begin{gathered}
\frac{d \chi(t)}{d t}=\frac{1}{2(1-\chi(t))}, \quad t>0, \\
\chi(0)=y_{1}^{0},
\end{gathered}
$$

from which it is easy to check that

$$
\begin{aligned}
& \chi(t)<1, \quad t \in\left[0,\left(y_{1}^{0}-1\right)^{2}\right), \\
& \chi(\cdot) \text { quenches at the time }\left(y_{1}^{0}-1\right)^{2} .
\end{aligned}
$$

Furthermore, making use of (28) and (29), we can derive, from the comparison theorem of ordinary differential equations, the following inequality:

$$
y_{1}(t) \geq \chi(t), \quad t \in\left[0, \min \left\{T_{q}\left(f^{(1)}, y^{0}, u\right),\left(y_{1}^{0}-1\right)^{2}\right\}\right) .
$$

This, combined with (27) and (30), implies (23) in Case 1.

Step 2. This is to prove (24) in Case 1.

Indeed, by (27) and (28), it is clear that $y_{1}(\cdot)$ is monotonously increasing over the interval $\left[0, T_{q}\left(f^{(1)}, y^{0}, u\right)\right)$. 
This, combined with (27), implies that $\lim _{t \rightarrow T_{q}\left(f^{(1)}, y^{0}, u\right)} y_{1}(t)$ exists and $\lim _{t \rightarrow T_{q}\left(f^{(1)}, y^{0}, u\right)} y_{1}(t) \leq 1$.

Now, we claim that

$$
\lim _{t \rightarrow T_{q}\left(f^{(1)}, y^{0}, u\right)} y_{1}(t)=1 .
$$

By contradiction, if $\lim _{t \rightarrow T_{q}\left(f^{(1)}, y^{0}, u\right)} y_{1}(t)=\beta<1$, then, by the continuity and the monotonicity of the solution $y_{1}(\cdot)$, it holds that $y_{1}(t) \leq \beta$ for $t \in\left[0, T_{q}\left(f^{(1)}, y^{0}, u\right)\right)$ and $y_{1}\left(T_{q}\left(f^{(1)}, y^{0}, u\right)\right)=\beta<1$. Then, we can extend the solution $y(\cdot)$ and can find an interval $\left[T_{q}\left(f^{(1)}, y^{0}, u\right), T_{q}\left(f^{(1)}, y^{0}, u\right)+\right.$ $\delta_{1}$ ) with $\delta_{1}$ sufficiently small such that $y_{1}(t)<1$ on the interval $\left[T_{q}\left(f^{(1)}, y^{0}, u\right), T_{q}\left(f^{(1)}, y^{0}, u\right)+\delta_{1}\right)$. However, $I\left(0, f^{(1)}, y^{0}, u\right)=\left[0, T_{q}\left(f^{(1)}, y^{0}, u\right)\right)$ and $I\left(0, f^{(1)}, y^{0}, u\right)$ is the maximal interval in which $y_{1}(t)<1$. This is a contradiction. Thus, (32) holds.

On the other hand, by (27), it is clear that

$$
\begin{aligned}
\left\|f^{(1)}(y(t))\right\| & \geq\left|\frac{y_{2}(t)}{1-y_{1}(t)}\right|>\frac{K_{0}+1 / K_{0}-1}{1-y_{1}(t)} \\
& \geq \frac{1}{1-y_{1}(t)}, \quad t \in\left[0, T_{q}\left(f^{(1)}, y^{0}, u\right)\right),
\end{aligned}
$$

from which and from (32), it holds that $\lim _{t \rightarrow T_{q}\left(f^{(1)}, y^{0}, u\right)}\left\|f^{(1)}(y(t))\right\|=+\infty$. This completes the proof of (24) in Case 1.

Step 3. This is to prove (25) in Case 1.

Since $y_{1}(t)<1$ for each $t \in\left[0, T_{q}\left(f^{(1)}, y^{0}, u\right)\right)$ and $y(\cdot)$ is continuous over the interval $\left[0, T_{q}\left(f^{(1)}, y^{0}, u\right)\right)$, we can get (25) in Case 1.

Step 4. This is to prove (26) in Case 1.

By system (4) with $f=f^{(1)}$, we conclude from (24), (27), and (5) that, for each $t \in\left[0, T_{q}\left(f^{(1)}, y^{0}, u\right)\right)$,

$$
\begin{aligned}
& -\frac{2}{3} \int_{t}^{T_{q}\left(f^{(1)}, y^{0}, u\right)} d\left(1-y_{1}(\tau)\right)^{3 / 2} \\
& =\frac{2}{3}\left(1-y_{1}(t)\right)^{3 / 2} \\
& =\int_{t}^{T_{q}\left(f^{(1)}, y^{0}, u\right)}\left(\frac{y_{2}(\tau)}{\left(1-y_{1}(\tau)\right)^{1 / 2}}\right. \\
& \left.\quad+\left(1-y_{1}(\tau)\right)^{1 / 2} b_{1}(\tau, u(\tau))\right) d \tau \\
& \geq \int_{t}^{T_{q}\left(f^{(1)}, y^{0}, u\right)}\left(\frac{1}{\left(1-1+1 / 2 K_{0}\right)^{1 / 2}}-\frac{K_{0}}{\sqrt{2 K_{0}}}\right) d \tau \\
& \geq\left(\sqrt{2}-\frac{1}{\sqrt{2}}\right) \sqrt{K_{0}}\left(T_{q}\left(f^{(1)}, y^{0}, u\right)-t\right),
\end{aligned}
$$

Case 2. $y^{0} \in S^{f^{(1)}}$ with $1<y_{1}^{0}<1+1 /\left(2 K_{0}\right)$ and $y_{2}^{0}>K_{0}+1$. We can use the very similar argument as that of Case 1 to give a proof of Case 2 (see [9] for the detailed proofs).

Remark 8. Let $y^{0} \in S^{f^{(1)}}$ and $u \in \mathscr{U}_{a d}$. Since $I\left(0, f^{(1)}, y^{0}, u\right)=$ $\left[0, T_{q}\left(f^{(1)}, y^{0}, u\right)\right)$, we can conclude from the definition of $I\left(0, f^{(1)}, y^{0}, u\right)$ that, for each $t \in\left[0, T_{q}\left(f^{(1)}, y^{0}, u\right)\right)$,

$$
\begin{aligned}
& y_{1}\left(t ; f^{(1)}, y^{0}, u\right)<1, \quad \text { when } y_{1}^{0}<1 \\
& y_{1}\left(t ; f^{(1)}, y^{0}, u\right)>1, \quad \text { when } y_{1}^{0}>1 .
\end{aligned}
$$

The following two lemmas play important roles in the proof of Theorem 1 with $f=f^{(2)}$ and $f=f^{(3)}$ and Theorem 3 (see [9] for the detailed proofs).

Lemma 9. For each $y^{0} \in S^{f^{(2)}}$ and each $u \in \mathscr{U}_{\text {ad }}$, there exists a time $T_{q}\left(f^{(2)}, y^{0}, u\right)$ in the interval $\left(0, T_{\max }\left(f^{(2)}, y^{0}, u\right)\right]$ holding the property that

$$
\begin{gathered}
T_{q}\left(f^{(2)}, y^{0}, u\right) \leq \frac{\left(2 K_{0}+1\right)\left(\left\|y^{0}\right\|-1\right)^{2}}{2 K_{0}}<+\infty, \\
\lim _{t \rightarrow T_{q}\left(f^{(2)}, y^{0}, u\right)}\left\|y\left(t ; f^{(2)}, y^{0}, u\right)\right\|=1, \\
\lim _{t \rightarrow T_{q}\left(f^{(2)}, y^{0}, u\right)}\left\|f^{(2)}\left(y\left(t ; f^{(2)}, y^{0}, u\right)\right)\right\|=+\infty, \\
\left\|f^{(2)}\left(y\left(t ; f^{(2)}, y^{0}, u\right)\right)\right\|<+\infty \quad \text { as } t \in\left[0, T_{q}\left(f^{(2)}, y^{0}, u\right)\right) .
\end{gathered}
$$

Moreover, there exists a positive constant $C$, independent of $y^{0} \in S^{f^{(2)}}$ and $u$, such that

$$
\begin{aligned}
& \frac{1}{\left|1-\left\|y\left(t ; f^{(2)}, y^{0}, u\right)\right\|\right|} \leq C\left(T_{q}\left(f^{(2)}, y^{0}, u\right)-t\right)^{-2 / 3} \\
& \text { for each } t \in\left[0, T_{q}\left(f^{(2)}, y^{0}, u\right)\right) .
\end{aligned}
$$

Lemma 10. For each $y^{0} \in S^{f^{(3)}}$ and each $u \in \mathcal{U}_{\text {ad }}$, there exists a time $T_{q}\left(f^{(3)}, y^{0}, u\right)$ in the interval $\left(0, T_{\max }\left(f^{(3)}, y^{0}, u\right)\right]$ holding the property that

$$
\begin{gathered}
T_{q}\left(f^{(3)}, y^{0}, u\right) \leq \frac{1}{4 K_{0}^{2}}<+\infty, \\
\lim _{t \rightarrow T_{q}\left(f^{(3)}, y^{0}, u\right)} y_{1}\left(t ; f^{(3)}, y^{0}, u\right) \\
=\lim _{t \rightarrow T_{q}\left(f^{(3)}, y^{0}, u\right)} y_{2}\left(t ; f^{(3)}, y^{0}, u\right)=1, \\
\lim _{t \rightarrow T_{q}\left(f^{(3)}, y^{0}, u\right)}\left\|f^{(3)}\left(y\left(t ; f^{(3)}, y^{0}, u\right)\right)\right\|=+\infty,
\end{gathered}
$$$$
\left\|f^{(3)}\left(y\left(t ; f^{(3)}, y^{0}, u\right)\right)\right\|<+\infty \quad \text { as } t \in\left[0, T_{q}\left(f^{(3)}, y^{0}, u\right)\right) .
$$

which implies (26) in Case 1. 
Moreover, there exists a positive constant $C$ depending on $y^{0} \in S^{f^{(3)}}$, but independent of $u$, such that, for each $t \in$ $\left[0, T_{q}\left(f^{(3)}, y^{0}, u\right)\right)$,

$$
\begin{aligned}
& \max \left\{\frac{1}{\left|1-y_{1}\left(t ; f^{(3)}, y^{0}, u\right)\right|}, \frac{1}{\left|1-y_{2}\left(t ; f^{(3)}, y^{0}, u\right)\right|}\right\} \\
& \quad \leq C\left(T_{q}\left(f^{(3)}, y^{0}, u\right)-t\right)^{-2 / 3} .
\end{aligned}
$$

2.3. Uniform Interval of Nonquenching. Let $[0, T]$ be a time interval. Given $f \in \Lambda, y^{0} \in S^{f}$ and $u \in \mathcal{U}_{\text {ad }}$, we say that the solution $y\left(\cdot ; f, y^{0}, u\right)$ does not quench on the interval $[0, T]$ if for each $t \in[0, T]$ we have

$$
\left\|f\left(y\left(t ; f, y^{0}, u\right)\right)\right\|<+\infty .
$$

Lemma 11. Suppose that $f \in \Lambda$ and $\left[0, t_{1}\right]$ is a closed interval. Assume $y^{0} \in S^{f}$. Let $u(\cdot)$ and $\left\{u_{k}(\cdot)\right\}_{k=1}^{\infty}$ be an element and a bounded sequence in $\mathcal{U}_{\text {ad }}$, respectively. Suppose that

$$
u_{k}(\tau) \rightarrow u(\tau) \quad \text { weakly star in } L^{\infty}\left(0, t_{1} ; \mathbb{R}^{2}\right)
$$

and the solution $y\left(\cdot ; f, y^{0}, u\right)$ does not quench on the interval $\left[0, t_{1}\right]$. Then there is a natural number $k_{0}$ such that, for each $k$ with $k \geq k_{0}$, the solution $y\left(\cdot ; f, y^{0}, u_{k}\right)$ does not quench on the interval $\left[0, t_{1}\right]$.

Using the lemmas we obtained in Sections 2.1 and 2.2, we can use the similar argument as we used in Lemma 2.2 of [8] to prove this lemma (see [9] for the detailed proofs.)

\section{Existence of Optimal Control}

Proof of Theorem 1. In this section, we will only give the proof of Theorem 1 in the case, where $y^{0} \in S^{f^{(1)}}$ with $1-1 /\left(2 K_{0}\right)<$ $y_{1}^{0}<1$ and $y_{2}^{0}>K_{0}+1 / K_{0}-1$. We can use very similar arguments to prove Theorem 1 in the cases, where $y^{0} \in S^{f^{(1)}}$ with $1<y_{1}^{0}<1+1 /\left(2 K_{0}\right), y_{2}^{0}>K_{0}+1, y^{0} \in S^{f^{(2)}}$, and $y^{0} \in S^{f^{(3)}}$.

Let $y^{0} \in S^{f^{(1)}}$ with $1-1 /\left(2 K_{0}\right)<y_{1}^{0}<1$ and $y_{2}^{0}>K_{0}+$ $1 / K_{0}-1$. By Lemma 7 and Remark 8 , it holds that, for each $u \in \mathcal{U}_{a d}, T_{q}\left(f^{(1)}, y^{0}, u\right)<+\infty$. Moreover,

$$
\lim _{\substack{t \rightarrow T_{q}\left(f^{(1)}, y^{0}, u\right) \\ y_{1}\left(t ; f^{(1)}, y^{0}, u\right)<1,}} y_{1}\left(t ; f^{(1)}, y^{0}, u\right)=1,
$$

Thus

$$
t^{*}=\inf _{u \in \mathscr{U}_{a d}} T_{q}\left(f^{(1)}, y^{0}, u\right)<+\infty
$$

Then, we can utilize the definitions of $t^{*}$ to get a sequence $\left\{u_{k}(\cdot)\right\}_{k=1}^{\infty}$ of controls in the set $\mathscr{U}_{a d}$ holding the following properties. (1) Each $t_{k}=: T_{q}\left(f^{(1)}, y^{0}, u_{k}\right)$ is a positive number; (2) $t_{1} \geq t_{2} \geq \cdots \geq t_{k} \cdots \geq t^{*}$ and $t_{k} \rightarrow t^{*}$ as $k \rightarrow+\infty$; (3) $\lim _{t \rightarrow t_{k}} y_{1}\left(t ; f^{(1)}, y^{0}, u_{k}\right)=1$ for all $k$.

For each $k$, write $y_{k}(\cdot)$ simply for $y\left(\cdot ; f^{(1)}, y^{0}, u_{k}\right)$. Now, we will complete the proof by the following two steps.

Step 1. This is to prove $t^{*}>0$.

Indeed, by Lemma 4 and Remark 8, we have

$$
1-\frac{1}{2 K_{0}}<y_{1 k}(t)<1 \quad \text { for each } k \text { and } t \in\left[0, t_{k}\right) .
$$

By system (4) with $f=f^{(1)}$, it holds that, for each $k$,

$$
\begin{aligned}
y_{2 k}(t)-y_{2}^{0}= & \int_{0}^{t}\left(y_{1 k}(\tau)+y_{2 k}(\tau)\right) d \tau \\
& +\int_{0}^{t} b_{2}\left(\tau, u_{k}(\tau)\right) d \tau, \quad t \in\left[0, t_{k}\right),
\end{aligned}
$$

from which and from (44) and (5), we get that, for each $k$,

$$
\left|y_{2 k}(t)\right| \leq\left|y_{2}^{0}\right|+C t_{k}+\int_{0}^{t}\left|y_{2 k}(\tau)\right| d \tau, \quad t \in\left[0, t_{k}\right) .
$$

Here and in what follows, $C$ is a positive constant independent of $k$ and $t$, which may be different in different context. Then, by the property (2) held by sequence $\left\{t_{k}\right\}$ and Gronwall's inequality, we derive, that for each $k$,

$$
\left|y_{2 k}(t)\right| \leq C, \quad t \in\left[0, t_{k}\right) .
$$

On the other hand, from system (4) with $f=f^{(1)},(47)$, (26) in Lemma 7, and (5), it holds that, for each $k$,

$$
\begin{aligned}
\left|y_{1 k}(t)-y_{1}^{0}\right| \leq & \int_{0}^{t_{k}}\left|\frac{y_{2 k}(\tau)}{1-y_{1 k}(\tau)}\right| d \tau \\
& +\int_{0}^{t_{k}}\left|b_{1}\left(\tau, u_{k}(\tau)\right)\right| d \tau \\
\leq & C \int_{0}^{t_{k}}\left(t_{k}-\tau\right)^{-2 / 3} d \tau+K_{0} t_{k} \\
\leq & C\left(t_{k}\right)^{1 / 3}+K_{0} t_{k}, \quad t \in\left[0, t_{k}\right) .
\end{aligned}
$$

If $t^{*}=0$, then, from the property (2) held by sequence $\left\{t_{k}\right\}$, we have the fact that the right side of the above inequality tends to 0 as $k \rightarrow+\infty$. This, together with the inequalities (48) and $y_{1}^{0}<1$, implies that we can find a natural number $K_{1}$ and a positive number $\beta_{0}$ such that $y_{1 K_{1}}(t) \leq y_{1}^{0}+\beta_{0}<1$ for all $t \in\left[0, t_{K_{1}}\right)$, which contradicts the property (3) held by sequence $\left\{t_{K_{1}}\right\}$. This completes the proof of Step 1 .

Step 2. This contains the existence of optimal control for the problem $(P)_{y^{0}}^{f^{(1)}}$.

Fix such a number $T$ such that $T>t^{*}$. It is clear that there exist a function $u^{*}$ in $L^{\infty}\left((0, T) ; \mathbb{R}^{2}\right)$ and a subsequence of the sequence $\left\{u_{k}(\cdot)\right\}_{k=1}^{\infty}$, still denoted in the same way, such that

$$
u_{k} \rightarrow u^{*} \quad \text { weakly star in } L^{\infty}\left(0, T ; \mathbb{R}^{2}\right) \text { as } k \longrightarrow+\infty \text {. }
$$


We extend the function $u^{*}$ by setting it to be zero on the interval $[T,+\infty)$ and denote the extension by $u^{*}$ again. Obviously, this extended function $u^{*}$ is in the set $\mathcal{U}_{a d}$.

Now, we will prove that $u^{*}$ is an optimal control for the problem $(P)_{y^{0}}^{f^{(1)}}$. We will carry out its proof by the following two claims.

Claim One. By the definition of $t^{*}$, it is obvious that the solution $y\left(\cdot ; f^{(1)}, y^{0}, u^{*}\right)$ does not quench at any time in the interval $\left[0, t^{*}\right)$.

Claim Two. $t^{*}=T_{q}\left(f^{(1)}, y^{0}, u^{*}\right)$. From the definition of $t^{*}$, it holds that $T_{q}\left(f^{(1)}, y^{0}, u^{*}\right) \geq t^{*}$. By seeking a contradiction, suppose that $t^{*}<T_{q}\left(f^{(1)}, y^{0}, u^{*}\right)$. Then we would find a number $\delta_{0}$ with $\left(t^{*}+\delta_{0}\right)<\min \left\{T, T_{q}\left(f^{(1)}, y^{0}, u^{*}\right)\right\}$ such that the solution $y\left(\cdot ; f^{(1)}, y^{0}, u^{*}\right)$ does not quench on the interval $\left[0, t^{*}+\delta_{0}\right]$. Thus, it follows from (49) and Lemma 11 that there exists a natural number $\widehat{k}$ such that, when $k \geq \widehat{k}$, the solution $y\left(\cdot ; f^{(1)}, y^{0}, u_{k}\right)$ does not quench on the interval $\left[0, t^{*}+\delta_{0}\right]$. Thus, $t_{k}=T_{q}\left(f^{(1)}, y^{0}, u_{k}\right)>t^{*}+\delta_{0}$, when $k \geq \widehat{k}$. Now, according to (26) in Lemma 7 , we can easily verify that, for all $k \geq \widehat{k}$,

$$
\begin{array}{r}
\frac{1}{1-y_{1}\left(t ; f^{(1)}, y^{0}, u_{k}\right)} \leq C\left(t_{k}-t\right)^{-2 / 3} \leq\left(\frac{\delta_{0}}{2}\right)^{-2 / 3}, \\
t \in\left[0, t^{*}+\frac{\delta_{0}}{2}\right],
\end{array}
$$

where $C$ is independent of $k$. This, together with the property (2) held by $\left\{t_{k}\right\}_{k=1}^{\infty}$, gives a positive constant $C$ independent of $k$ such that $1 /\left(1-y_{1}\left(t_{k} ; f^{(1)}, y^{0}, u_{k}\right)\right) \leq C$, for all $k \geq \widehat{k}$. This contradicts with the property (3) by $\left\{t_{k}\right\}_{k=1}^{\infty}$.

Thus, we have completed the proof of Theorem 1 .

\section{Proof of Pontryagin Maximum Principle}

Proof of Theorem 2. We will only give the proof of Theorem 2 in the case, where $y^{0} \in S^{f^{(1)}}$ with $1-1 /\left(2 K_{0}\right)<y_{1}^{0}<1$ and $y_{2}^{0}>K_{0}+1 / K_{0}-1$. We can use very similar arguments to prove Theorem 2 in the case, where $y^{0} \in S^{f^{(1)}}$ with $1<y_{1}^{0}<$ $1+1 /\left(2 K_{0}\right)$ and $y_{2}^{0}>K_{0}+1$.

Let $y^{0} \in S^{f^{(1)}}$ with $1-1 /\left(2 K_{0}\right)<y_{1}^{0}<1$ and $y_{2}^{0}>K_{0}+$ $1 / K_{0}-1$. We will prove the theorem in a series of steps as follows.

Step 1. This is to set up a penalty functional and to study the related properties.

Since the number $t^{*}$ is the optimal time for the problem $(P)_{y^{0}}^{f^{(1)}}$, the solution $y\left(\cdot ; f^{(1)}, y^{0}, u\right)$, corresponding to each $u$ in the set $\mathcal{U}_{a d}$, does not quench on $\left[0, t^{*}-\varepsilon\right]$, for any $\varepsilon \in$ $\left(0, t^{*}\right)$. Moreover, $T_{q}\left(f^{(1)}, y^{0}, u\right) \geq t^{*}$ for all $u \in \mathcal{U}_{\text {ad }}$. Let $T^{*}$ be a fixed number such that $T^{*}>t^{*}$. Write $\mathscr{U}\left[0, T^{*}\right]$ for the set $\left\{\left.u\right|_{\left[0, T^{*}\right]} ; u \in \mathcal{U}_{a d}\right\}$. We introduce the Ekeland distance $d^{*}$ over the set $\mathscr{U}\left[0, T^{*}\right]$ by setting

$$
\begin{array}{r}
d^{*}(u, v)=\operatorname{meas}\left(\left\{t \in\left[0, T^{*}\right] ; u(t) \neq v(t)\right\}\right) \\
\forall u, v \in \mathcal{U}_{a d} .
\end{array}
$$

Here and in what follows, meas $(E)$ stands for the Lebesgue measure of a measurable set $E$ in $\mathbb{R}^{1}$. Then $\left(\mathcal{U}\left[0, T^{*}\right], d^{*}\right)$ forms a completed metric space (see [5], page 145). For each $\varepsilon \in\left(0, t^{*}\right)$, we define a penalty functional $J_{\varepsilon}$ : $\left(\mathcal{U}\left[0, T^{*}\right], d^{*}\right) \rightarrow R^{+}$by setting

$$
J_{\varepsilon}(u(\cdot))=\frac{\left|y_{1}\left(t^{*}-\varepsilon ; f^{(1)}, y^{0}, u\right)-1\right|^{2}}{2} .
$$

We claim that $J_{\varepsilon}$ is continuous over the space $\left(\mathcal{U}\left[0, T^{*}\right], d^{*}\right)$. Before moving forward to the proof of this claim, we make the following observation, which will be often used in what follows. Since $f_{1}^{(1)}(y)=y_{2} /\left(1-y_{1}\right), f_{2}^{(1)}(y)=y_{1}+y_{2}$, and $y=\left(y_{1}, y_{2}\right)^{T} \in \mathbb{R}^{2}$ with $y_{1} \neq 1$, it holds that, for each $y=$ $\left(y_{1}, y_{2}\right)^{T} \in \mathbb{R}^{2}$ with $y_{1} \neq 1$,

$$
f_{y}^{(1)}(y)=\left(\begin{array}{cc}
\frac{y_{2}}{\left(1-y_{1}\right)^{2}} & 1 \\
\frac{1}{1-y_{1}} & 1
\end{array}\right) \text {. }
$$

Now, we come back to prove the above claim. Let $v$ be an element and let $\left\{u_{k}(\cdot)\right\}_{k=1}^{\infty}$ be a sequence in the space $\mathcal{U}\left[0, T^{*}\right]$ such that $d^{*}\left(u_{k}, v\right) \rightarrow 0$ as $k \rightarrow+\infty$. Then, it is clear that $u_{k} \rightarrow v$ strongly in $L^{1}\left(\left(0, T^{*}\right) ; \mathbb{R}^{2}\right)$ as $k \rightarrow+\infty$. Throughout this proof, we will write $y(\cdot)$ simply for $y\left(\cdot ; f^{(1)}, y^{0}, v\right)$ and, for each $k$, we will write $y_{k}(\cdot)$ simply for $y\left(\cdot ; f^{(1)}, y^{0}, u_{k}\right)$; $C$ is a constant independent of $k$ and $t$, which may be different in different context. Since $T_{q}\left(f^{(1)}, y^{0}, u\right) \geq t^{*}$ for all $u \in$ $\mathcal{U}\left[0, T^{*}\right]$, it holds from the system (4) with $f=f^{(1)}$ that

$$
\begin{aligned}
& \left|y_{1 k}(t)-y_{1}(t)\right|+\left|y_{2 k}(t)-y_{2}(t)\right| \\
& \leq \int_{0}^{t}\left(\left|\frac{y_{2 k}(\tau)}{1-y_{1 k}(\tau)}-\frac{y_{2}(\tau)}{1-y_{1}(\tau)}\right|+\left|y_{1 k}(\tau)-y_{1}(\tau)\right|\right. \\
& \left.\quad+\left|y_{2 k}(\tau)-y_{2}(\tau)\right|\right) d \tau \\
& +C \int_{0}^{t}\left\|B(\tau) u_{k}(\tau)-B(\tau) v(\tau)\right\| d \tau \\
& \quad t \in\left[0, t^{*}-\varepsilon\right]
\end{aligned}
$$

From Lemmas 4 and 7 and Remark 8, we obtain that, for each $t \in\left[0, T_{q}\left(f^{(1)}, y^{0}, v\right)\right)$,

$$
\begin{gathered}
1-\frac{1}{2 K_{0}}<y_{1}(t)<1, \\
\frac{1}{1-y_{1}(t)} \leq C\left(T_{q}\left(f^{(1)}, y^{0}, v\right)-t\right)^{-2 / 3} ;
\end{gathered}
$$


for all $k$ and for each $t \in\left[0, T_{q}\left(f^{(1)}, y^{0}, u_{k}\right)\right)$,

$$
\begin{gathered}
1-\frac{1}{2 K_{0}}<y_{1 k}(t)<1, \\
\frac{1}{1-y_{1 k}(t)} \leq C\left(T_{q}\left(f^{(1)}, y^{0}, u_{k}\right)-t\right)^{-2 / 3} .
\end{gathered}
$$

By the property that $T\left(f^{(1)}, y^{0}, u\right) \geq t^{*}$ for all $u \in \mathscr{U}\left[0, T^{*}\right]$ again, (55), and (56), it holds that

$$
\begin{aligned}
\frac{1}{1-y_{1}(t)} \leq C \varepsilon^{-2 / 3}, \quad \frac{1}{1-y_{1 k}(t)} \leq C \varepsilon^{-2 / 3} \\
\text { for each } k \text { and } t \in\left[0, t^{*}-\varepsilon\right] .
\end{aligned}
$$

On the other hand, because $y(\cdot)$ is continuous over $\left[0, t^{*}-\right.$ $\varepsilon]$, we get $\left|y_{1}(t)\right| \leq C$ and $\left|y_{2}(t)\right| \leq C, t \in\left[0, t^{*}-\varepsilon\right]$. This, together with (54), (55), (56), and (57), implies that, for each $k$ and $t \in\left[0, t^{*}-\varepsilon\right]$,

$$
\begin{aligned}
& \left|y_{1 k}(t)-y_{1}(t)\right|+\left|y_{2 k}(t)-y_{2}(t)\right| \\
& \leq C \int_{0}^{t}\left(\left|y_{1 k}(\tau)-y_{1}(\tau)\right|+\left|y_{2 k}(\tau)-y_{2}(\tau)\right|\right) d \tau \\
& \quad+C \int_{0}^{t^{*}-\varepsilon}\left\|B(\tau) u_{k}(\tau)-B(\tau) v(\tau)\right\| d \tau .
\end{aligned}
$$

Now, we can apply Gronwall's inequality to get that, as $k \rightarrow+\infty$,

$$
\begin{array}{r}
\left|y_{1 k}(t)-y_{1}(t)\right|+\left|y_{2 k}(t)-y_{2}(t)\right| \longrightarrow 0 \\
\text { uniformly in } t \in\left[0, t^{*}-\varepsilon\right] .
\end{array}
$$

Hence, we have proved the continuity of the functional $J_{\varepsilon}$.

Step 2. This is to apply the Ekeland variational principle.

It is clear that

$$
\begin{gathered}
J_{\mathcal{\varepsilon}}\left(u^{*}(\cdot)\right)=\frac{\left|y_{1}\left(t^{*}-\varepsilon ; f^{(1)}, y^{0}, u^{*}\right)-1\right|^{2}}{2} \\
=: \sigma(\varepsilon) \longrightarrow 0 \quad \text { as } \varepsilon \longrightarrow 0^{+}, \\
J_{\mathcal{\varepsilon}}\left(u^{*}(\cdot)\right) \leq \inf _{u \in \mathscr{U}\left[0, T^{*}\right]} J_{\mathcal{\varepsilon}}(u(\cdot))+\sigma(\varepsilon) \quad \text { for each } \varepsilon \in\left(0, t^{*}\right) .
\end{gathered}
$$

Then, we can utilize Ekeland's variational principle (see, for instance, [5], page 136-137) to find a control $u^{\varepsilon}(\cdot) \in \mathscr{U}\left[0, T^{*}\right]$ enjoying the following properties:

$$
\begin{gathered}
d^{*}\left(u^{*}, u^{\varepsilon}\right) \leq \sqrt{\sigma(\varepsilon)}, \\
-\sqrt{\sigma(\varepsilon)} d^{*}\left(v, u^{\varepsilon}\right) \leq J_{\varepsilon}(v(\cdot))-J_{\varepsilon}\left(u^{\varepsilon}(\cdot)\right) \\
\forall v(\cdot) \in \mathscr{U}\left[0, T^{*}\right] .
\end{gathered}
$$

Let $u(\cdot) \in \mathcal{U}\left[0, T^{*}\right]$. By the variant of the Lyapunov theorem (see, for instance, [5], Chapter 4), we can get, corresponding to each $\rho \in(0,1)$, a measurable set $E_{\rho, \varepsilon}$ in the interval $\left[0, T^{*}\right]$ such that meas $\left(E_{\rho, \varepsilon}\right)=\rho T^{*}$ and

$$
\begin{aligned}
& \left\|\int_{E_{\rho, \varepsilon} \cap[0, t]} B(\tau)\left(u-u^{\varepsilon}\right)(\tau) d \tau-\rho \int_{0}^{t} B(\tau)\left(u-u^{\varepsilon}\right)(\tau) d \tau\right\| \\
& \quad \leq \rho^{2}, \quad t \in\left[0, T^{*}\right] .
\end{aligned}
$$

Now, we construct the following spike function of $u^{\varepsilon}$ with respect to $u$ by setting

$$
u_{\rho}^{\varepsilon}(t)= \begin{cases}u^{\varepsilon}(t), & t \in\left[0, T^{*}\right] \backslash E_{\rho, \varepsilon}, \\ u(t), & t \in E_{\rho, \varepsilon} .\end{cases}
$$

It is obvious that the control $u_{\rho}^{\varepsilon}(\cdot)$ is in $\mathscr{U}\left[0, T^{*}\right]$. Write $y_{\rho}^{\varepsilon}(\cdot)$ and $y^{\varepsilon}(\cdot)$ simply for the solutions $y\left(\cdot ; f^{(1)}, y^{0}, u_{\rho}^{\varepsilon}\right)$ and $y\left(\cdot ; f^{(1)}, y^{0}, u^{\varepsilon}\right)$, respectively. Clearly, they do not quench on the interval $\left[0, t^{*}-\varepsilon\right]$. Set $z_{\rho}^{\varepsilon}(t)=\left(y_{\rho}^{\varepsilon}(t)-y^{\varepsilon}(t)\right) / \rho, t \in$ $\left[0, t^{*}-\varepsilon\right]$. Then, for each $t \in\left[0, t^{*}-\varepsilon\right]$, it holds that

$$
\begin{aligned}
z_{\rho}^{\varepsilon}(t)= & \int_{0}^{t} \int_{0}^{1}\left[f_{y}^{(1)}\left(y^{\varepsilon}+\theta\left(y_{\rho}^{\varepsilon}-y^{\varepsilon}\right)\right)\right]^{T}(\tau) d \theta z_{\rho}^{\varepsilon}(\tau) d \tau \\
& +\int_{0}^{t} \frac{B(\tau)\left[u_{\rho}^{\varepsilon}(\tau)-u^{\varepsilon}(\tau)\right]}{\rho} d \tau .
\end{aligned}
$$

Step 3. This is to show the uniform convergence of the family $\left\{z_{\rho}^{\varepsilon}(\cdot)\right\}_{\rho>0}$ on the interval $\left[0, t^{*}-\varepsilon\right]$ for $\rho \rightarrow 0^{+}$.

It follows from (63) that $d^{*}\left(u_{\rho}^{\varepsilon}, u^{\varepsilon}\right) \rightarrow 0$ as $\rho \rightarrow 0^{+}$. Thus, we can use the same argument in the proof of (59) to get

$$
y_{\rho}^{\varepsilon}(\cdot) \longrightarrow y^{\varepsilon}(\cdot) \quad \text { uniformly on }\left[0, t^{*}-\varepsilon\right] \text { as } \rho \longrightarrow 0^{+} .
$$

On the other hand, making use of (62) and (63), it holds that, for all $t \in\left[0, t^{*}-\varepsilon\right]$,

$$
\begin{aligned}
& \int_{0}^{t} \frac{B(\tau)\left[u_{\rho}^{\varepsilon}(\tau)-u^{\varepsilon}(\tau)\right]}{\rho} d \tau \\
& \quad=\int_{0}^{t} B(\tau)\left(u(\tau)-u^{\varepsilon}(\tau)\right) d \tau+\frac{\left\|r_{\rho}^{\varepsilon}(t)\right\|}{\rho} .
\end{aligned}
$$

Here, the function $r_{\rho}^{\varepsilon}(t)$ has the property $\left\|r_{\rho}^{\varepsilon}(t)\right\| \leq \rho^{2}$ for all $t \in\left[0, t^{*}-\varepsilon\right]$.

Let $z^{\varepsilon}(\cdot)$ be the unique solution to the following system:

$$
\begin{gathered}
\frac{d z^{\varepsilon}(t)}{d t}=\left[f_{y}^{(1)}\left(y^{\varepsilon}(t)\right)\right]^{T} z^{\varepsilon}(t)+B(t)\left(u(t)-u^{\varepsilon}(t)\right), \\
t \in\left[0, t^{*}-\varepsilon\right], \\
z^{\varepsilon}(0)=0 .
\end{gathered}
$$


Then, by (64) and (67), we get that, for each $t \in\left[0, t^{*}-\varepsilon\right]$,

$$
\begin{aligned}
& \left\|z_{\rho}^{\varepsilon}(t)-z^{\varepsilon}(t)\right\| \\
& \leq \int_{0}^{t}\left\|\int_{0}^{1}\left[f_{y}^{(1)}\left(y^{\varepsilon}+\theta\left(y_{\rho}^{\varepsilon}-y^{\varepsilon}\right)\right)\right]^{T}(\tau) d \theta\right\| \\
& \times\left\|\left(z_{\rho}^{\varepsilon}(\tau)-z^{\varepsilon}(\tau)\right)\right\| d \tau \\
& +\int_{0}^{t} \| \int_{0}^{1}\left[f_{y}^{(1)}\left(y^{\varepsilon}+\theta\left(y_{\rho}^{\varepsilon}-y^{\varepsilon}\right)\right)\right]^{T}(\tau) d \theta \\
& \quad \| \int_{0}^{t} \frac{B(\tau)\left[u_{\rho}^{\varepsilon}(\tau)-u^{\varepsilon}(\tau)\right]}{\rho} d \tau \\
& \quad-\int_{0}^{t} B(\tau)\left(u(\tau)-u^{\varepsilon}(\tau)\right) d \tau \| .
\end{aligned}
$$

Corresponding to each $t \in\left[0, t^{*}-\varepsilon\right]$, write $m_{\rho}(t)$ for the sum of the last two terms in the right hand of (68).

Clearly, it follows from (65) and (66) that $m_{\rho}(\cdot) \rightarrow 0$ uniformly on $\left[0, t^{*}-\varepsilon\right]$ as $\rho \rightarrow 0^{+}$.

Since $y^{\varepsilon}(\cdot)$ is continuous and $1-1 /\left(2 K_{0}\right)<y_{1}^{\varepsilon}(t)<1$ for all $t \in\left[0, t^{*}-\varepsilon\right]$ (see Lemma 4 and Remark 8 ), there exists a constant $\widehat{C}$ with $0<\widehat{C}<1$ such that $y_{1}^{\varepsilon}(t)<\widehat{C}$ for each $t \epsilon$ $\left[0, t^{*}-\varepsilon\right]$. Then, it follows from (65) that, when $\rho$ is sufficiently small, we have $y_{1 \rho}^{\varepsilon}(t)<\widehat{C}$ for all $t \in\left[0, t^{*}-\varepsilon\right]$. Hence, when $\rho$ is sufficiently small, it holds that, for each $t \in\left[0, t^{*}-\varepsilon\right]$,

$$
\begin{gathered}
\frac{1}{1-\left\{y_{1}^{\varepsilon}(t)+\theta\left(y_{1 \rho}^{\varepsilon}(t)-y_{1}^{\varepsilon}(t)\right)\right\}} \leq \frac{1}{1-\widehat{C}}, \\
\frac{1}{\left\{1-\left[y_{1}^{\varepsilon}(t)+\theta\left(y_{1 \rho}^{\varepsilon}(t)-y_{1}^{\varepsilon}(t)\right)\right]\right\}^{2}} \leq \frac{1}{(1-\widehat{C})^{2}} .
\end{gathered}
$$

On the other hand, from the continuity of $y^{\varepsilon}(\cdot)$ and (65), we get that, when $\rho$ is sufficiently small,

$$
\left|y_{2}^{\varepsilon}(t)+\theta\left(y_{2 \rho}^{\varepsilon}(t)-y_{2}^{\varepsilon}(t)\right)\right| \leq C, \quad t \in\left[0, t^{*}-\varepsilon\right] .
$$

Here and in what follows, $C$ is a constant independent of $\rho$ and $t$, which may be different in different context. From the above inequality, (53), and (69), it follows that, when $\rho$ is sufficiently small,

$$
\left\|\int_{0}^{1}\left[f_{y}^{(1)}\left(y^{\varepsilon}+\theta\left(y_{\rho}^{\varepsilon}-y^{\varepsilon}\right)\right)\right]^{T}(\tau) d \theta\right\| \leq C, \quad t \in\left[0, t^{*}-\varepsilon\right],
$$

from which and from (68), it holds from the Gronwall's inequality that, when $\rho$ is sufficiently small,

$$
\left\|z_{\rho}^{\varepsilon}(t)-z^{\varepsilon}(t)\right\| \leq m_{\rho}(t)+C \int_{0}^{t} m_{\rho}(\tau) d \tau, \quad t \in\left[0, t^{*}-\varepsilon\right] .
$$

Hence, it holds that $z_{\rho}^{\varepsilon}(\cdot) \rightarrow z^{\varepsilon}(\cdot)$ uniformly on $\left[0, t^{*}-\varepsilon\right]$ as $\rho \rightarrow 0^{+}$, from which, we obtain that

$$
y_{\rho}^{\varepsilon}(t)=y^{\varepsilon}(t)+\rho z^{\varepsilon}(t)+o(\rho) \text { uniformly on }\left[0, t^{*}-\varepsilon\right]
$$

Step 4. This is to get certain necessary conditions for the control $u^{\varepsilon}$.

By the second inequality of (61) and according to the definition of the functional $J_{\varepsilon}$, we can easily verify the following inequality:

$$
-\sqrt{\sigma(\varepsilon)} T^{*} \leq \frac{\left|y_{1 \rho}^{\varepsilon}\left(t^{*}-\varepsilon\right)-1\right|^{2} / 2-\left|y_{1}^{\varepsilon}\left(t^{*}-\varepsilon\right)-1\right|^{2} / 2}{\rho} .
$$

This, together with (65) and (73), implies that

$$
\begin{aligned}
- & \sqrt{\sigma(\varepsilon)} T^{*} \\
& \leq \frac{\lim _{\rho \rightarrow 0^{+}}\left[\left|y_{1 \rho}^{\varepsilon}\left(t^{*}-\varepsilon\right)-1\right|^{2} / 2-\left|y_{1}^{\varepsilon}\left(t^{*}-\varepsilon\right)-1\right|^{2} / 2\right]}{\rho} \\
& =\left(y_{1}^{\varepsilon}\left(t^{*}-\varepsilon\right)-1\right) z_{1}^{\varepsilon}\left(t^{*}-\varepsilon\right) .
\end{aligned}
$$

Let $\psi^{\varepsilon}(\cdot)$ be the unique solution for the dual system:

$$
\begin{array}{cc}
\frac{d \psi^{\varepsilon}(t)}{d t}=-f_{y}^{(1)}\left(y^{\varepsilon}(t)\right) \psi^{\varepsilon}(t), & t \in\left[0, t^{*}-\varepsilon\right], \\
\psi_{1}^{\varepsilon}\left(t^{*}-\varepsilon\right)=1-y_{1}^{\varepsilon}\left(t^{*}-\varepsilon\right), & \psi_{2}^{\varepsilon}\left(t^{*}-\varepsilon\right)=0 .
\end{array}
$$

Then, it follows from (75), (67), and (76) that the following inequality yields

$$
\int_{0}^{t^{*}-\varepsilon}\left\langle\psi^{\varepsilon}(\tau), B(\tau)\left(u(\tau)-u^{\varepsilon}(\tau)\right)\right\rangle d \tau \leq \sqrt{\sigma(\varepsilon)} T^{*}
$$

Step 5. This is to obtain a uniform estimate for $\psi^{\varepsilon}(\cdot)$ with $\varepsilon>0$ sufficiently small.

Since $\psi^{\varepsilon}(\cdot)$ solves $(76)$, we see that

$$
\begin{array}{r}
\psi^{\varepsilon}(t)=\psi^{\varepsilon}\left(t^{*}-\varepsilon\right)+\int_{t}^{t^{*}-\varepsilon} f_{y}^{(1)}\left(y^{\varepsilon}(\tau)\right) \psi^{\varepsilon}(\tau) d \tau \\
t \in\left[0, t^{*}-\varepsilon\right] .
\end{array}
$$

By Lemma 4 and Remark 8, it holds that

$$
\begin{array}{r}
1-\frac{1}{2 K_{0}}<y_{1}^{\varepsilon}(t)<1, \quad y_{2}^{\varepsilon}(t)>K_{0}+\frac{1}{K_{0}}-1 \geq 1, \\
t \in\left[0, t^{*}-\varepsilon\right] .
\end{array}
$$


This, combined with (53), shows that, for each $t \in\left[0, t^{*}-\varepsilon\right]$,

$$
\begin{aligned}
\left\|\psi^{\varepsilon}(t)\right\| \leq & \left(1-y_{1}^{\varepsilon}\left(t^{*}-\varepsilon\right)\right) \\
& +\int_{t}^{t^{*}-\varepsilon}\left\|f_{y}^{(1)}\left(y^{\varepsilon}(\tau)\right)\right\|\left\|\psi^{\varepsilon}(\tau)\right\| d \tau \\
\leq & \left(1-y_{1}^{\varepsilon}\left(t^{*}-\varepsilon\right)\right) \\
& +\int_{t}^{t^{*}-\varepsilon}\left(\frac{y_{2}^{\varepsilon}(\tau)}{\left(1-y_{1}^{\varepsilon}(\tau)\right)^{2}}+\frac{1}{1-y_{1}^{\varepsilon}(\tau)}+2\right) \\
& \times\left\|\psi^{\varepsilon}(\tau)\right\| d \tau .
\end{aligned}
$$

Now we can apply the Gronwall's inequality to get that, for all $t \in\left[0, t^{*}-\varepsilon\right]$,

$$
\begin{aligned}
& \left\|\psi^{\varepsilon}(t)\right\| \\
& \leq\left(1-y_{1}^{\varepsilon}\left(t^{*}-\varepsilon\right)\right) \\
& \quad \times \exp \left\{\int_{t}^{t^{*}-\varepsilon}\left(\frac{y_{2}^{\varepsilon}(\tau)}{\left(1-y_{1}^{\varepsilon}(\tau)\right)^{2}}+\frac{1}{1-y_{1}^{\varepsilon}(\tau)}+2\right) d \tau\right\} .
\end{aligned}
$$

On the other hand, according to the system satisfied by $y^{\varepsilon}(\cdot)$, it follows that, for every $t \in\left[0, t^{*}-\varepsilon\right]$,

$$
\begin{aligned}
& \int_{t}^{t^{*}-\varepsilon} \frac{d\left(1-y_{1}^{\varepsilon}(\tau)\right)}{1-y_{1}^{\varepsilon}(\tau)}=\ln \frac{1-y_{1}^{\varepsilon}\left(t^{*}-\varepsilon\right)}{1-y_{1}^{\varepsilon}(t)} \\
&=\int_{t}^{t^{*}-\varepsilon}-\frac{1}{1-y_{1}^{\varepsilon}(\tau)}\left(\frac{y_{2}^{\varepsilon}(\tau)}{1-y_{1}^{\varepsilon}(\tau)}\right. \\
&\left.+b_{1}\left(\tau, u^{\varepsilon}(\tau)\right)\right) d \tau ;
\end{aligned}
$$

namely, we have the equation as follows:

$$
\begin{aligned}
& 1-y_{1}^{\varepsilon}\left(t^{*}-\varepsilon\right)=\left(1-y_{1}^{\varepsilon}(t)\right) \\
& \cdot \exp \left\{\int_{t}^{t^{*}-\varepsilon}-\frac{y_{2}^{\varepsilon}(\tau)}{\left(1-y_{1}^{\varepsilon}(\tau)\right)^{2}} d \tau\right\} \\
& \cdot \exp \left\{\int_{t}^{t^{*}-\varepsilon} \frac{-b_{1}\left(\tau, u^{\varepsilon}(\tau)\right)}{1-y_{1}^{\varepsilon}(\tau)} d \tau\right\}, \\
& t \in\left[0, t^{*}-\varepsilon\right] .
\end{aligned}
$$

Then, making use of the above equation and by (81), we obtain

$$
\begin{aligned}
& \left\|\psi^{\varepsilon}(t)\right\| \\
& \leq\left(1-y_{1}^{\varepsilon}(t)\right) \\
& \times \exp \left\{\int_{t}^{t^{*}-\varepsilon}\left(\frac{1}{1-y_{1}^{\varepsilon}(\tau)}+2-\frac{b_{1}\left(\tau, u^{\varepsilon}(\tau)\right)}{1-y_{1}^{\varepsilon}(\tau)}\right) d \tau\right\}, \\
& t \in\left[0, t^{*}-\varepsilon\right] .
\end{aligned}
$$

On the other hand, by Lemma 7 and the inequality $T_{q}\left(f^{(1)}, y^{0}, u^{\varepsilon}\right) \geq t^{*}$, we get

$$
\frac{1}{1-y_{1}^{\varepsilon}(t)} \leq C\left(t^{*}-t\right)^{-2 / 3} \quad \text { for each } t \in\left[0, t^{*}-\varepsilon\right],
$$

where $C$ is independent of $\varepsilon \in\left(0, t^{*}\right)$ and $t$. This, together with (84) and (5), implies that

$$
\left\|\psi^{\varepsilon}(t)\right\| \leq C_{1}\left(1-y_{1}^{\varepsilon}(t)\right), \quad t \in\left[0, t^{*}-\varepsilon\right],
$$

where $C_{1}$ is independent of $\varepsilon \in\left(0, t^{*}\right)$ and $t$.

Step 6. This contains the convergence of a subsequence of the family $\left\{\psi^{\varepsilon}(\cdot)\right\}_{\varepsilon>0}$.

First of all, corresponding to each $\varepsilon \in\left(0, t^{*}\right)$, we extend the function $\psi^{\varepsilon}(\cdot)$ by setting $\psi_{1}^{\varepsilon}(\cdot)$ to be $1-y_{1}^{\varepsilon}\left(t^{*}-\varepsilon\right)$ on $\left(t^{*}-\varepsilon, t^{*}\right]$ and setting $\psi_{2}^{\varepsilon}(\cdot)$ to be 0 on $\left(t^{*}-\varepsilon, t^{*}\right]$ and denote the extended function by $\psi^{\varepsilon}(\cdot)$ again. Clearly, this extended function is continuous on $\left[0, t^{*}\right]$.

Now, we take a sequence $\left\{\delta_{m}\right\}_{m=1}^{\infty}$ of numbers from the interval $\left(0, t^{*}\right)$ such that (i) $\lim _{m \rightarrow+\infty} \delta_{m}=0$ and (ii) $\delta_{1}>$ $\delta_{2}>\cdots$. Corresponding to the number $\delta_{1}$, we can take a sequence $\left\{\varepsilon_{n}\right\}_{n=1}^{\infty}$ from the set $\{\varepsilon\}_{0<\varepsilon<t^{*}}$ such that $\lim _{n \rightarrow+\infty} \varepsilon_{n}=$ 0 and $\left[0, t^{*}-\delta_{1}\right] \subset\left[0, t^{*}-\varepsilon_{n}\right]$ for all $n=1,2, \ldots$.

By (79), the sequence $\left\{1-y_{1}^{\varepsilon_{n}}(\cdot)\right\}_{n=1}^{\infty}$ is uniformly bounded on the interval $\left[0, t^{*}-\delta_{1}\right]$. This, together with (86), implies that $\left\{\psi^{\varepsilon_{n}}(\cdot)\right\}_{n=1}^{\infty}$ is uniformly bounded on $\left[0, t^{*}-\delta_{1}\right]$.

On the other hand, by system (4) with $f=f^{(1)}$, we can use the similar argument we used to prove (47) to conclude that

$$
\left|y_{2}^{\varepsilon_{n}}(t)\right| \leq C, \quad t \in\left[0, t^{*}-\delta_{1}\right]
$$

where $C$ is independent of $n$ and $t$.

Next, we will prove that the sequence $\left\{\psi^{\varepsilon_{n}}(\cdot)\right\}_{n=1}^{\infty}$ is equicontinuous on $\left[0, t^{*}-\delta_{1}\right]$.

Indeed, for each $s_{1}$ and $s_{2}$ in the interval $\left[0, t^{*}-\delta_{1}\right]$ with $s_{1}<s_{2}$, it follows from (76), (53), and (86) that

$$
\begin{aligned}
& \left|\psi^{\varepsilon_{n}}\left(s_{1}\right)-\psi^{\varepsilon_{n}}\left(s_{2}\right)\right| \\
& \leq \int_{s_{1}}^{s_{2}}\left\|f_{y}^{(1)}\left(y^{\varepsilon_{n}}(t)\right)\right\|\left\|\psi^{\varepsilon_{n}}(t)\right\| d t \\
& \leq C_{1} \int_{s_{1}}^{s_{2}}\left(\frac{y_{2}^{\varepsilon_{n}}(t)}{\left(1-y_{1}^{\varepsilon_{n}}(t)\right)^{2}}+\frac{1}{1-y_{1}^{\varepsilon_{n}}(t)}+2\right)\left(1-y_{1}^{\varepsilon_{n}}(t)\right) d t,
\end{aligned}
$$

from which and from (79), (85), and (87), it holds that

$$
\begin{aligned}
\left|\psi^{\varepsilon_{n}}\left(s_{1}\right)-\psi^{\varepsilon_{n}}\left(s_{2}\right)\right| \leq C \int_{s_{1}}^{s_{2}}\left(\frac{1}{1-y_{1}^{\varepsilon_{n}}(t)}+1\right) d t \\
\leq C \int_{s_{1}}^{s_{2}}\left(t^{*}-t\right)^{-2 / 3} d t+C\left(s_{2}-s_{1}\right) \\
\leq C\left[\left(t^{*}-s_{1}\right)^{1 / 3}-\left(t^{*}-s_{2}\right)^{1 / 3}\right] \\
\\
+C\left(s_{2}-s_{1}\right),
\end{aligned}
$$


where $C$ is independent of $n, s_{1}$, and $s_{2}$. This implies that the sequence $\left\{\psi^{\varepsilon_{n}}(\cdot)\right\}_{n=1}^{\infty}$ is equicontinuous on $\left[0, t^{*}-\delta_{1}\right]$. Hence, we can utilize the Arzela-Ascoli theorem and the standard diagonal argument to see that there exists a subsequence $\left\{\psi^{\varepsilon_{n, n}}(\cdot)\right\}$ which is uniformly convergent on $\left[0, t^{*}-\delta\right]$ for each $\delta \in\left(0, t^{*}\right)$.

Let $\psi(t)=\lim _{n \rightarrow+\infty} \psi^{\varepsilon_{n, n}}(t), t \in\left[0, t^{*}\right)$. Then it holds that

$$
\begin{gathered}
\psi^{\varepsilon_{n, n}}(\cdot) \longrightarrow \psi(\cdot) \text { uniformly on }\left[0, t^{*}-\delta\right] \\
\text { as } n \longrightarrow+\infty, \quad \text { for each } \delta \in\left(0, t^{*}\right) .
\end{gathered}
$$

Step 7. This is to extend the function $\psi$ over the interval $\left[0, t^{*}\right]$.

Since all solutions, $y^{\varepsilon_{n, n}}(\cdot), n=1,2, \ldots$, do not quench on every interval $\left[0, t^{*}-\delta\right]$ with $\delta \in\left(0, t^{*}\right)$ and $\lim _{n \rightarrow+\infty} d^{*}\left(u^{\varepsilon_{n, n}}, u^{*}\right)=0$, we can use the same arguments in the proof of (59) to get

$$
\begin{array}{r}
y^{\varepsilon_{n, n}}(\cdot) \longrightarrow y^{*}(\cdot) \text { uniformly on }\left[0, t^{*}-\delta\right] \\
\text { as } n \longrightarrow+\infty, \quad \text { for each } \delta \in\left(0, t^{*}\right) .
\end{array}
$$

This, together with (86) and (90), yields the following inequality:

$$
\|\psi(t)\| \leq C_{1}\left(1-y_{1}^{*}(t)\right) \quad \forall t \in\left[0, t^{*}\right) .
$$

Since the constant $C_{1}$ is independent of $t$, it holds that

$$
0 \leq \lim _{t \rightarrow t^{*}}\|\psi(t)\| \leq \lim _{t \rightarrow t^{*}} C_{1}\left(1-y_{1}^{*}(t)\right) .
$$

On the other hand, because $t^{*}=T_{q}\left(f^{(1)}, y^{0}, u^{*}\right)$, we conclude from Lemma 7 that $\lim _{t \rightarrow t^{*}} y_{1}^{*}(t)=1$, from which and from (93), we obtain that $\lim _{t \rightarrow t^{*}} \psi(t)=0$. Now, we extend the function $\psi(\cdot)$ by setting it to be zero at the time $t^{*}$ and still denote the extension by $\psi(\cdot)$. Clearly, this extended function $\psi(\cdot)$ is continuous on the interval $\left[0, t^{*}\right]$ and has the property $\psi\left(t^{*}\right)=0$.

Step 8 . This is to verify that the function $\psi(\cdot)$ solves the system (10).

Clearly, the function $\psi^{\varepsilon_{n, n}}(\cdot)$ holds the following property:

$$
\begin{aligned}
\psi^{\varepsilon_{n, n}}(t)= & \psi^{\varepsilon_{n, n}}\left(t^{*}-\varepsilon_{n, n}\right) \\
& +\int_{t}^{t^{*}-\varepsilon_{n, n}} f_{y}^{(1)}\left(y^{\varepsilon_{n, n}}(\tau)\right) \psi^{\varepsilon_{n, n}}(\tau) d \tau, \\
& \quad t \in\left[0, t^{*}-\varepsilon_{n, n}\right],
\end{aligned}
$$

where $\psi_{1}^{\varepsilon_{n, n}}\left(t^{*}-\varepsilon_{n, n}\right)=1-y_{1}^{\varepsilon_{n, n}}\left(t^{*}-\varepsilon_{n, n}\right)$ and $\psi_{2}^{\varepsilon_{n, n}}\left(t^{*}-\varepsilon_{n, n}\right)=0$. We first claim that

$$
\frac{1}{1-y_{1}^{\varepsilon_{n, n}}\left(t^{*}-\varepsilon_{n, n}\right)} \longrightarrow+\infty,
$$

or equivalently, $\psi^{\varepsilon_{n, n}}\left(t^{*}-\varepsilon_{n, n}\right) \longrightarrow 0$ as $n \longrightarrow+\infty$.

If (95) were not true, then there would exist a positive constant $\beta$ such that

$$
\frac{1}{1-y_{1}^{\varepsilon_{n, n}}\left(t^{*}-\varepsilon_{n, n}\right)} \leq \beta \quad \text { for infinitely many } n .
$$

On the other hand, we take a number $\gamma_{1}$ with $1-1 /\left(2 K_{0}\right)<$ $\gamma_{1}<1$ and $1 /\left(1-\gamma_{1}\right)>\beta$. Because $\lim _{t \rightarrow t^{*}} y_{1}^{*}(t)=1$, we can find a number $\delta \in\left(0, t^{*}\right)$ such that $y_{1}^{*}\left(t^{*}-\delta\right)>\gamma_{1}$. Since $\lim _{n \rightarrow+\infty} \varepsilon_{n, n}=0$, there is a natural number $N_{1}$ such that

$$
\left(t^{*}-\varepsilon_{n, n}\right)>\left(t^{*}-\delta\right) \quad \forall n \geq N_{1} .
$$

Now, we can utilize Lemma 4 to get

$$
\frac{1}{1-y_{1}^{\varepsilon_{n, n}}\left(t^{*}-\varepsilon_{n, n}\right)} \geq \frac{1}{1-\gamma_{1}}>\beta \quad \forall n \geq N_{1},
$$

which contradicts with (96). Therefore, we have proved (95).

Next, we claim that

$$
\begin{aligned}
& \lim _{n \rightarrow+\infty} \int_{t}^{t^{*}-\varepsilon_{n, n}} f_{y}^{(1)}\left(y^{\varepsilon_{n, n}}(\tau)\right) \psi^{\varepsilon_{n, n}}(\tau) d \tau \\
& =\int_{t}^{t^{*}} f_{y}^{(1)}\left(y^{*}(\tau)\right) \psi(\tau) d \tau, \quad t \in\left[0, t^{*}\right) .
\end{aligned}
$$

Corresponding to each $n$, we define a function $F_{n}(\cdot)$ by setting

$$
F_{n}(t)= \begin{cases}f_{y}^{(1)}\left(y^{\varepsilon_{n, n}}(t)\right) \psi^{\varepsilon_{n, n}}(t), & t \in\left[0, t^{*}-\varepsilon_{n, n}\right], \\ 0, & t \in\left(t^{*}-\varepsilon_{n, n}, t^{*}\right] .\end{cases}
$$

It is clear that all functions, $F_{n}(\cdot), n=1,2, \ldots$, are measurable on the interval $\left[0, t^{*}\right]$. We will first give an estimate on the sequence $\left\{\left\|F_{n}(\cdot)\right\|\right\}_{n=1}^{\infty}$. Let $t \in\left[0, t^{*}\right]$. In the case that $n$ is such that $t \in\left[0, t^{*}-\varepsilon_{n, n}\right]$, by (53), (79), (85), (86), and (87), we have the following estimate:

$$
\begin{aligned}
&\left\|F_{n}(t)\right\|=\left\|f_{y}^{(1)}\left(y^{\varepsilon_{n, n}}(t)\right) \psi^{\varepsilon_{n, n}}(t)\right\| \\
& \leq C\left(\frac{\left|y_{2}^{\varepsilon_{n, n}}(t)\right|}{\left(1-y_{1}^{\varepsilon_{n, n}}(t)\right)^{2}}+\frac{1}{1-y_{1}^{\varepsilon_{n, n}}(t)}+2\right) \\
& \times\left(1-y_{1}^{\varepsilon_{n, n}}(t)\right) \leq C+C\left(t^{*}-t\right)^{-2 / 3}, \\
& t \in\left[0, t^{*}-\varepsilon_{n, n}\right],
\end{aligned}
$$

where $C$ is independent of $n$ and $t$.

On the other hand, if $n$ is such that $t \in\left(t^{*}-\varepsilon_{n, n}, t^{*}\right]$, then $F_{n}(t)=0$. This, together with (101), implies that

$$
\begin{array}{r}
\left\|F_{n}(t)\right\| \leq C+C\left(t^{*}-t\right)^{-2 / 3}, \quad \forall t \in\left[0, t^{*}\right], \\
\forall n=1,2, \ldots .
\end{array}
$$

Then, we are going to show that the sequence $\left\{F_{n}(t)\right\}_{n=1}^{\infty}$ is convergent for each $t \in\left[0, t^{*}\right)$. Indeed, corresponding to each $t \in\left[0, t^{*}\right)$, there exists a natural number $N_{2}$ such that $t \in\left[0, t^{*}-\varepsilon_{n, n}\right]$ when $n \geq N_{2}$. Then, by (90), (91), and (102), we can apply Lebesgue dominated convergence theorem to get

$$
\lim _{n \rightarrow+\infty} \int_{t}^{t^{*}} F_{n}(\tau) d \tau=\int_{t}^{t^{*}} f_{y}^{(1)}\left(y^{*}(\tau)\right) \psi(\tau) d \tau,
$$

$$
\text { for each } t \in\left[0, t^{*}\right) \text {, }
$$

from which, (99) follows immediately. 
Now, let $s \in\left[0, t^{*}\right)$. Clearly, it holds that $s \in\left[0, t^{*}-\varepsilon_{n, n}\right)$ for $n$ sufficiently large. Thus, making use of (95) and (99), we can pass to the limit for $n \rightarrow+\infty$ in (94), where $t=s$, to get

$$
\psi(s)=\int_{s}^{t^{*}} f_{y}^{(1)}\left(y^{*}(\tau)\right) \psi(\tau) d \tau .
$$

Since $s$ can be arbitrarily taken from $\left[0, t^{*}\right)$ in the above equation, we already hold the first equation of (10). On the other hand, we proved that $\psi\left(t^{*}\right)=0$ in the end of Step 7 . Hence, the function $\psi(\cdot)$ solves the system (10).

Step 9. This is to prove (11).

We can use the similar argument we used in the proof of Step 9 of Theorem 1.2 in [8] to prove (11).

Step 10 . This is to show the nontriviality of the function $\psi(\cdot)$.

By the equations satisfied by $y^{\varepsilon_{n, n}}(\cdot)$ and $\psi^{\varepsilon_{n, n}}(\cdot)$, respectively, we see that, for each $t \in\left[0, t^{*}-\varepsilon_{n, n}\right]$,

$$
\begin{aligned}
& \int_{t}^{t^{*}-\varepsilon_{n, n}} \frac{d}{d \tau}\left(\frac{\psi_{1}^{\varepsilon_{n, n}}(\tau)}{1-y_{1}^{\varepsilon_{n, n}}(\tau)}\right) d \tau \\
& =\int_{t}^{t^{*}-\varepsilon_{n, n}} \frac{-\psi_{2}^{\varepsilon_{n, n}}(\tau)}{1-y_{1}^{\varepsilon_{n, n}}(\tau)} d \tau \\
& \quad+\int_{t}^{t^{*}-\varepsilon_{n, n}} \frac{\psi_{1}^{\varepsilon_{n, n}}(\tau)}{\left(1-y_{1}^{\varepsilon_{n, n}}(\tau)\right)^{2}} b_{1}\left(\tau, u^{\varepsilon_{n, n}}(\tau)\right) d \tau .
\end{aligned}
$$

Because $\left\{\psi_{1}^{\varepsilon_{n, n}}\left(t^{*}-\varepsilon_{n, n}\right)\right\} /\left\{1-y_{1}^{\varepsilon_{n, n}}\left(t-\varepsilon_{n, n}\right)\right\}=1$, it follows from (105) that, for each $t \in\left[0, t^{*}-\varepsilon_{n, n}\right]$,

$$
\begin{aligned}
1-\frac{\psi_{1}^{\varepsilon_{n, n}}(t)}{1-y_{1}^{\varepsilon_{n, n}}(t)}= & \int_{t}^{t^{*}-\varepsilon_{n, n}} \frac{-\psi_{2}^{\varepsilon_{n, n}}(\tau)}{1-y_{1}^{\varepsilon_{n, n}}(\tau)} d \tau \\
& +\int_{t}^{t^{*}-\varepsilon_{n, n}} \frac{\psi_{1}^{\varepsilon_{n, n}}(\tau)}{\left(1-y_{1}^{\varepsilon_{n, n}}(\tau)\right)^{2}} \\
& \quad \times b_{1}\left(\tau, u^{\varepsilon_{n, n}}(\tau)\right) d \tau .
\end{aligned}
$$

By (86), we can make use of the very similar arguments as those in the proof of (99) to verify that

$$
\begin{aligned}
& \lim _{n \rightarrow+\infty}\left(\int_{t}^{t^{*}-\varepsilon_{n, n}} \frac{-\psi_{2}^{\varepsilon_{n, n}}(\tau)}{1-y_{1}^{\varepsilon_{n, n}}(\tau)} d \tau\right. \\
& \left.\quad+\int_{t}^{t^{*}-\varepsilon_{n, n}} \frac{\psi_{1}^{\varepsilon_{n, n}}(\tau)}{\left(1-y_{1}^{\varepsilon_{n, n}}(\tau)\right)^{2}} b_{1}\left(\tau, u^{\varepsilon_{n, n}}(\tau)\right) d \tau\right) \\
& =\int_{t}^{t^{*}} \frac{-\psi_{2}(\tau)}{1-y_{1}^{*}(\tau)} d \tau+\int_{t}^{t^{*}} \frac{\psi_{1}(\tau)}{\left(1-y_{1}^{*}(\tau)\right)^{2}} b_{1}\left(\tau, u^{*}(\tau)\right) d \tau,
\end{aligned}
$$

for each $t \in\left[0, t^{*}\right)$, which, together with (90), (91), and (106), implies that, for each $t \in\left[0, t^{*}\right)$,

$$
\begin{aligned}
1-\frac{\psi_{1}(t)}{1-y_{1}^{*}(t)}= & \int_{t}^{t^{*}} \frac{-\psi_{2}(\tau)}{1-y_{1}^{*}(\tau)} d \tau \\
& +\int_{t}^{t^{*}} \frac{\psi_{1}(\tau)}{\left(1-y_{1}^{*}(\tau)\right)^{2}} b_{1}\left(\tau, u^{*}(\tau)\right) d \tau .
\end{aligned}
$$

This shows that the function $\psi(\cdot)$ is not trivial.

Step 11. This is to prove (12).

Indeed, by (23) in Lemma 7, (12) holds.

Thus, we complete the proof of Theorem 2 in the case, where $y^{0} \in S^{f^{(1)}}$ with $1-1 /\left(2 K_{0}\right)<y_{1}^{0}<1$ and $y_{2}^{0}>$ $K_{0}+1 / K_{0}-1$.

We can use similar arguments in the proof of Theorem 2 to prove Theorem 3 (see [9] for the detailed proofs).

\section{Conflict of Interests}

The author declares that there is no conflict of interests regarding the publication of this paper.

\section{Acknowledgments}

This work is Supported by the National Natural Science Foundation of China (no. 11071036) and the National Basis Research Program of China (973 Program no. 2011CB808002).

\section{References}

[1] H. Kawarada, "On solutions of initial-boundary problem for $u_{t}=u_{x x}+1 /(1-u)$, , Publications of the Research Institute for Mathematical Sciences, vol. 10, no. 3, pp. 729-736, 1975.

[2] C. Y. Chan and H. G. Kaper, "Quenching for semilinear singular parabolic problems," SIAM Journal on Mathematical Analysis, vol. 20, no. 3, pp. 558-566, 1989.

[3] V. Barbu, Analysis and Control of Nonlinear Infinite-Dimensional Systems, vol. 190, Academic Press, Boston, Mass, USA, 1993.

[4] H. O. Fattorini, Infinite Dimensional Linear Control Systems. The Time Optimal and Norm Optimal Problems, vol. 201, Elsevier Science, Amsterdam, The Netherlands, 2005.

[5] X. J. Li and J. M. Yong, Optimal Control Theory for InfiniteDimensional Systems, Birkhäuser, Boston, Mass, USA, 1995.

[6] S. Luan, H. Gao, and X. Li, "Optimal control problem for an elliptic equation which has exactly two solutions," Optimal Control Applications \& Methods, vol. 32, no. 6, pp. 734-747, 2011.

[7] G. Zheng and B. Ma, "A time optimal control problem of some linear switching controlled ordinary differential equations," Advances in Difference Equations, vol. 2012, article 52, 7 pages, 2012.

[8] P. Lin and G. Wang, "Blowup time optimal control for ordinary differential equations," SIAM Journal on Control and Optimization, vol. 49, no. 1, pp. 73-105, 2011.

[9] P. Lin, "Quenching time optimal control for some ordinary differential equations," http://arxiv.org/abs/1209.0784. In press. 


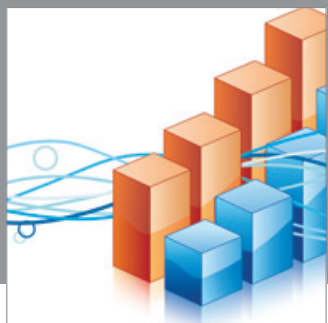

Advances in

Operations Research

mansans

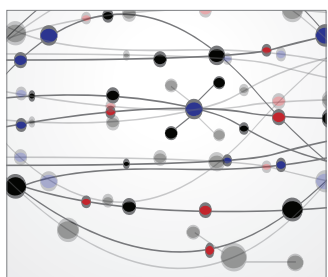

The Scientific World Journal
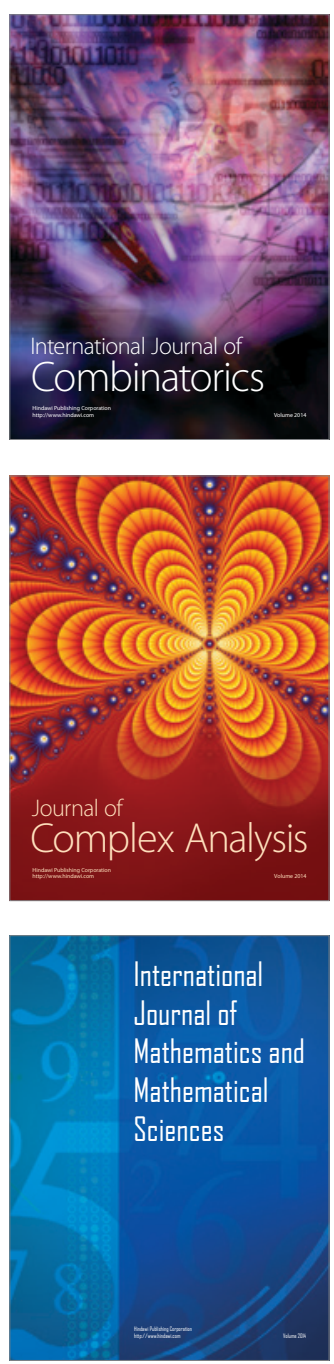
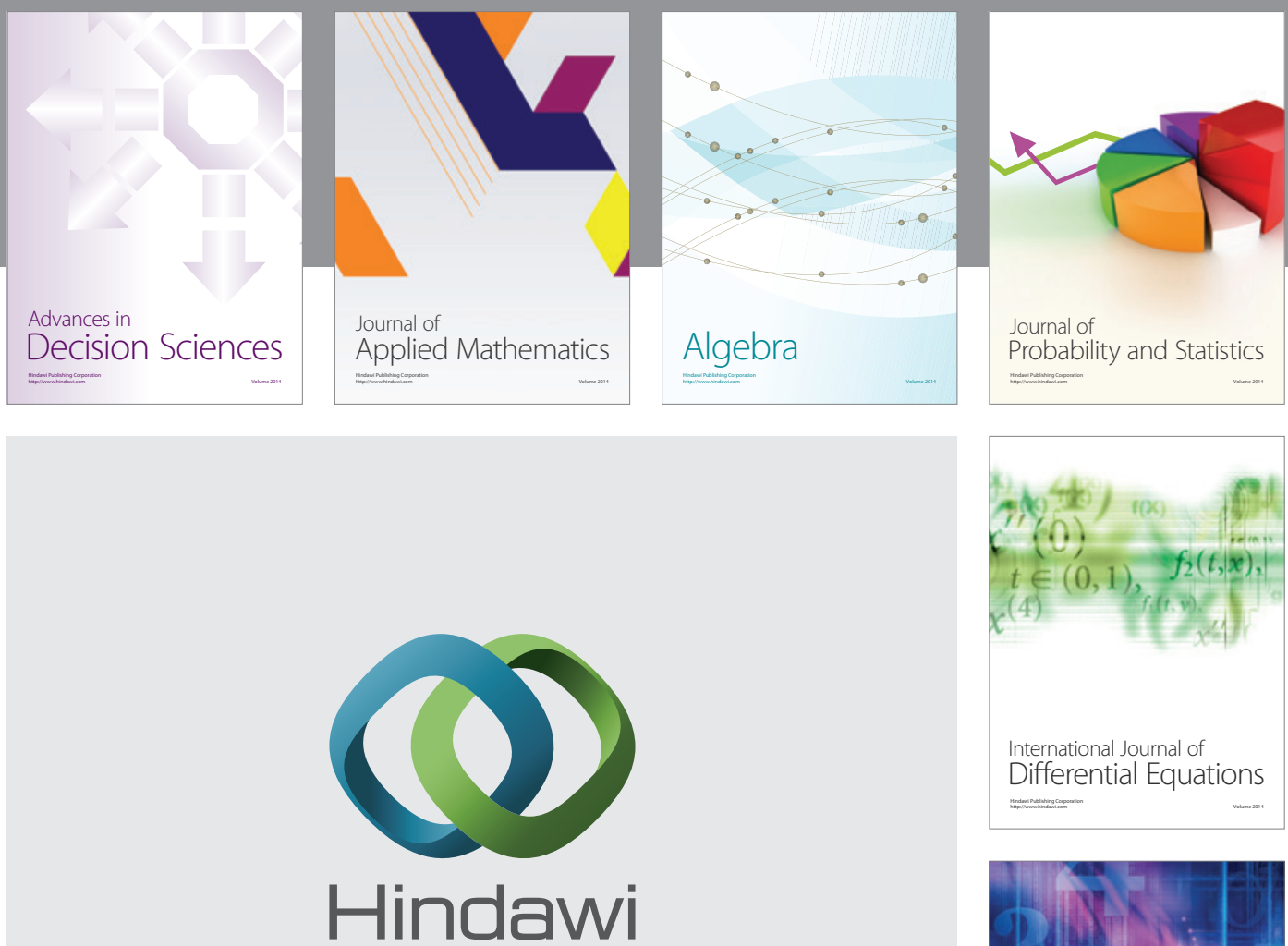

Submit your manuscripts at http://www.hindawi.com
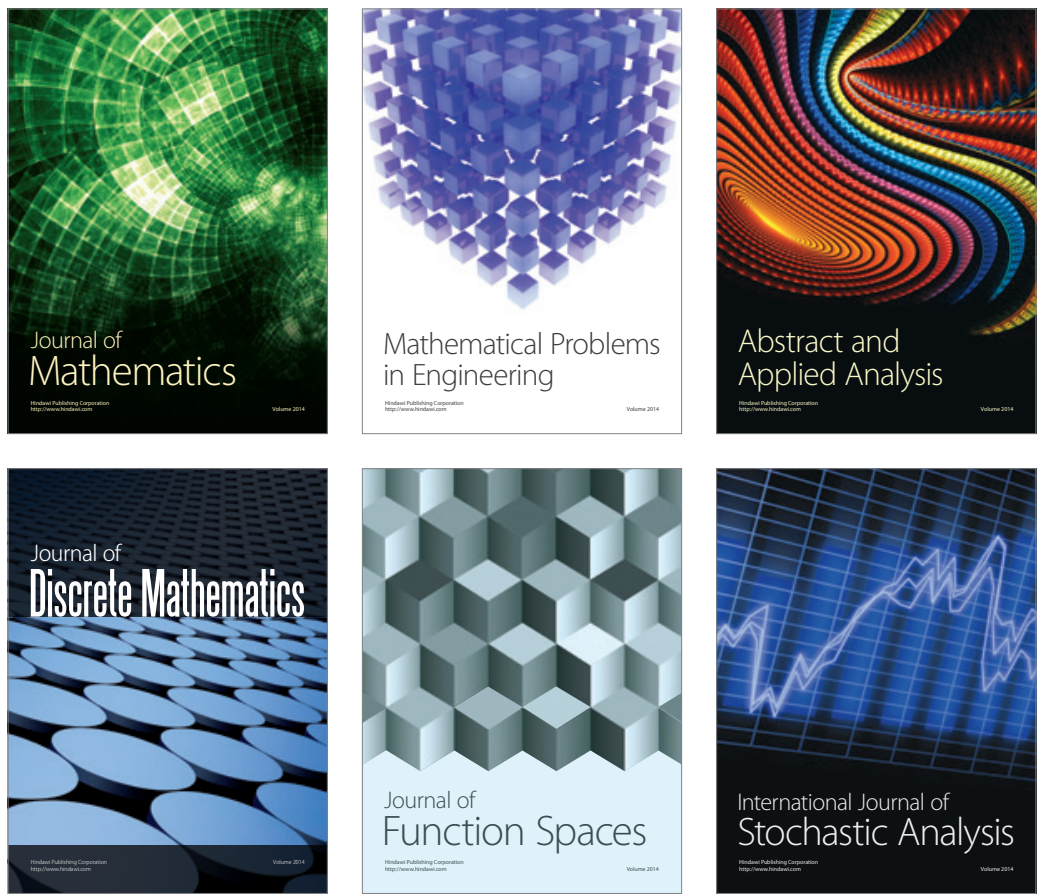

Journal of

Function Spaces

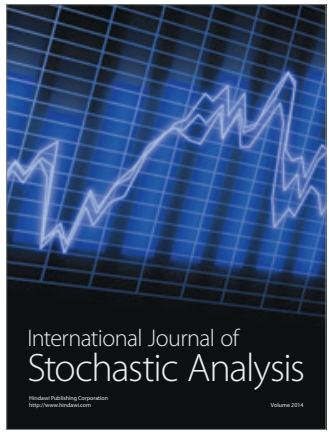

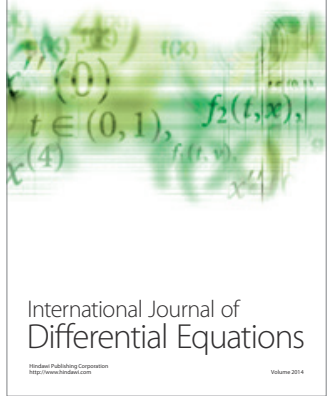
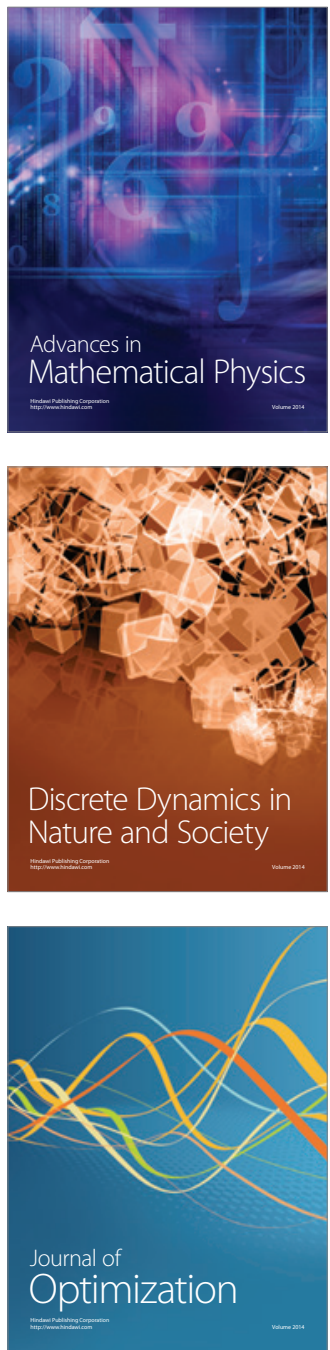